\title{
Meandering of the Spiral Tip An Alternative Approach
}

\author{
Martin Golubitsky \\ Department of Mathematics \\ University of Houston \\ Houston, TX 77204-3476 \\ USA
}

\author{
Victor G. LeBlanc \\ Department of Mathematics \\ University of Ottawa \\ Ottawa, ON K1N 6N5 \\ CANADA
}

\author{
Ian Melbourne \\ Department of Mathematics \\ University of Houston \\ Houston, TX 77204-3476 \\ USA
}

February 10, 1997

\begin{abstract}
Meandering of a one-armed spiral tip has been noted in chemical reactions and numerical simulations. Barkley, Kness and Tuckerman show that meandering can begin by Hopf bifurcation from a rigidly rotating spiral wave (a point that is verified in a B-Z reaction by Li, Ouyang, Petrov and Swinney). At the codimension two point where (in an appropriate sense) the frequency at Hopf bifurcation equals the frequency of the spiral wave, Barkley notes that spiral tip meandering can turn to linearly translating spiral tip motion.

Barkley also presents a model showing that the linear motion of the spiral tip is a resonance phenomenon, and this point is verified experimentally by $\mathrm{Li}$ et al. and proved rigorously by Wulff. In this paper we suggest an alternative development of Barkley's model extending the center bundle constructions of Krupa from compact groups to noncompact groups and from finite dimensions to function spaces. Our
\end{abstract}



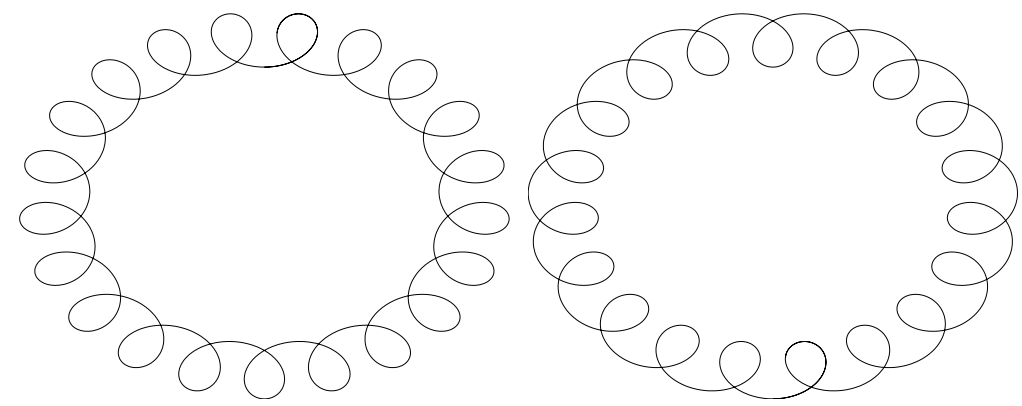

Figure 1: Epicycle motion of spiral tip: outward and inward petals.

reduction works only under certain simplifying assumptions which are not valid for Euclidean group actions. Recent work of Sandstede, Scheel and Wulff shows how to overcome these difficulties.

This approach allows us to consider various bifurcations from a rotating wave. In particular, we analyze the codimension two Barkley bifurcation and the codimension two Takens-Bogdanov bifurcation from a rotating wave. We also discuss Hopf bifurcation from a many armed spiral showing that meandering and resonant linear motion of the spiral tip do not always occur.

\section{Introduction}

Spiral waves have been observed both in experiments [20, 13, 14] and numerical simulations $[13,4]$. See Kapral and Showalter [11] for descriptions of recent work on spiral waves and for additional references. In our discussion we focus on one particular aspect of spiral wave theory - the observation by Barkley [3] that linear meandering of the spiral tip is caused by Euclidean symmetry.

Planar spirals rigidly rotate and, as a result, the tip of the spiral traces out a circle in the plane. Winfree [20] observed that under certain circumstances the tip of a spiral can meander and create flower-like movements as in Figure 1. These motions are quasiperiodic two frequency motions, which can be thought of as an epicycle motion superimposed on the basic spiral wave circle. When the motion on the epicycle is in the same orientation as the motion on the circle (either clockwise or counterclockwise), then the petals of the flowers point in; when the motions have the opposite orientation, the petals point out. Winfree observed both types of quasiperiodic motions and the possibility of changing the directions of the petals — which we call a change in petality - as a system parameter is varied. 
The epicycle motion can be written as

$$
q(t)=e^{i \omega_{1} t}\left(z_{1}+e^{-i \omega_{2} t} z_{2}\right)
$$

where $z_{1} \in \mathbf{R}$ and $z_{2} \in \mathbf{C}$. In these coordinates the change in petality occurs when $\omega_{1}=\omega_{2}$. We note that in order to see well-defined petals the ratio of the amplitudes $\left|z_{2}\right| /\left|z_{1}\right|$ should be large. Nevertheless, these quasiperiodic states can be formed, as Barkley et al. [4] observed in numerical simulations of a reaction-diffusion system, through a Hopf bifurcation from the rotating spiral wave. That observation has been confirmed in recent chemical wave experiments by Li et al. [14].

In the epicycle motion (1.1), Hopf bifurcation corresponds to the secondary amplitude $z_{2}=0$. From the standard bifurcation theory point of view, there is nothing significant about Hopf bifurcation at this critical parameter value where $\omega_{1}=\omega_{2}$. However, in Barkley's numerical simulation [1] and in experiments such as those by Li et al. [14] another phenomenon is observed. As the change in petality is approached, the radius of the second frequency $\left|z_{2}\right|$ grows unboundedly large. In particular, at the point of petality change, the spiral tip appears to drift in a straight line off to infinity. See Figure 2. Thus, unbounded growth of the second frequency amplitude is a feature that seems to be connected with change in petality.

As we mentioned, for changes in petality to be observed, the amplitude of the epicycle should be large, while near Hopf bifurcation points, this same amplitude must be small. This dichotomy suggests that standard Hopf bifurcation by itself cannot provide an explanation for petality change and unbounded growth. However, Barkley [3] made the keen observation that Euclidean symmetry coupled with Hopf bifurcation is behind the unbounded growth that accompanies changes in petality. The basis of his argument turns out, in retrospect, to be quite simple.

Suppose we consider a reaction-diffusion system on the unbounded plane. Such systems of equations have Euclidean symmetry. Suppose the system has a spiral wave solution and that the time-periodic spiral wave undergoes a Hopf bifurcation to a quasiperiodic motion. At the point of Hopf bifurcation, symmetry forces (at least) five eigenvalues of the linearized system to be on the imaginary axis - two generated from Hopf bifurcation and three generated by Euclidean symmetry. Assuming that center manifold ideas hold, the time evolution of the meandering spiral (and hence the time evolution of the meandering spiral tip) is described by a five dimensional Euclidean equivariant system of ODEs. There are three variables of this system representing the Euclidean group - the translation variable $p \in \mathbf{R}^{2} \cong \mathbf{C}$ and the rotation variable $\varphi \in \mathbf{S}^{1}$ - and the variable $q \in \mathbf{C}$ representing the amplitude of the eigenfunction of Hopf bifurcation. 


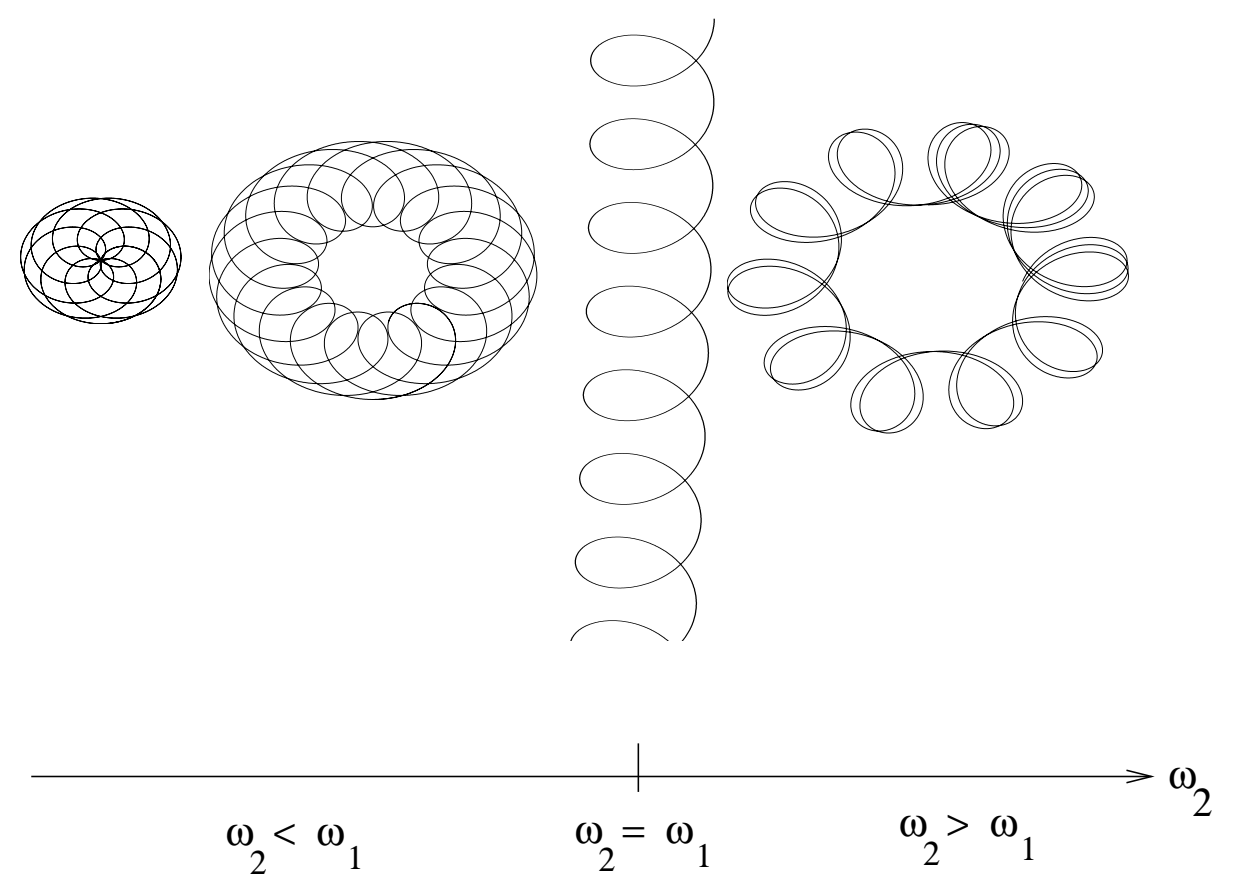

Figure 2: Growth of flower near change in petality: path of $\int_{0}^{t} q(s) d s$, where $q(t)$ is as in (1.1) with $\omega_{1}=1, z_{1}=1, z_{2}=0.3$ and $\omega_{2}=0.61,0.85,1,1.11$.

In these variables Barkley [3] assumes that the translation symmetry acts by

$$
T_{x}(p, \varphi, q)=(p+x, \varphi, q) .
$$

It follows that the ODE vector field is independent of $p$ and that the $(\varphi, q)$ equations decouple, see Lemmas 3.6 and 4.1 in this paper. Suppose that a quasiperiodic solution is found in the $(\varphi, q)$ equations. Then $p$ is obtained by integration. To understand how this integration causes unbounded growth in $p$, rewrite (1.1) as

$$
q(t)=e^{i \omega_{1} t} z_{1}+e^{i\left(\omega_{1}-\omega_{2}\right) t} z_{2} .
$$

Then, for some constant $C \in \mathbf{R}$,

$$
p(t)=\int_{0}^{t} q(\tau) d \tau= \begin{cases}\frac{1}{i}\left(\frac{z_{1}}{\omega_{1}} e^{i \omega_{1} t}+\frac{z_{2}}{\omega_{1}-\omega_{2}} e^{i\left(\omega_{1}-\omega_{2}\right) t}\right)+C & \omega_{1} \neq \omega_{2} \\ \frac{z_{1}}{i \omega_{1}} e^{i \omega_{1} t}+z_{2} t+C & \omega_{1}=\omega_{2} .\end{cases}
$$


It follows that if $z_{2} \neq 0$, then as $\omega_{2}$ approaches $\omega_{1}$ a resonant blow-up in $p(t)$ occurs. In particular, when $\omega_{2}-\omega_{1} \neq 0$ is small the amplitude of the second term is large, the motion of the second term is on a circle of large radius, and the motion of $p(t)$ is a small perturbation of this circular motion. (The center of this circle is determined by $C$.) Thus, this blow-up is the source of the unbounded growth of the second frequency mode and occurs even when the magnitude of $z_{2}$ is small.

Barkley [2] performed a numerical linear stability analysis for the basic time-periodic spiral wave solution and showed that there is a Hopf bifurcation. In particular, a simple pair of eigenvalues were shown to cross the imaginary axis while three neutral eigenvalues lie on the imaginary axis and the remainder of the spectrum is bounded into the left-half plane. Starting from Barkley's numerical calculation, Wulff [21], in a major mathematical work on spirals, has given a rigorous proof that resonant unbounded growth occurs in Hopf bifurcation near the codimension two point where $\omega_{1}=\omega_{2}$. Wulff approaches the study of this Hopf bifurcation using Liapunov-Schmidt reduction. Her proof is nontrivial, as there are technical difficulties, such as the nonsmoothness of the group action, which must be overcome.

In this paper we suggest an alternative to the methods in [21], which we believe helps in the understanding of the work of Barkley and Wulff. Our approach to bifurcations from rotating waves in Euclidean equivariant differential equations extends Krupa's ideas [12] of bifurcation from relative equilibria. Krupa's methods lead to the construction of and reduction to a center bundle over the critical group orbit. We note that Biktashev et al. [5] obtain a similar reduction for the case of one-armed spirals by considering an orbit space reduction. For many armed spirals, the center bundle reduction has the advantage of not introducing singularities. Indeed, we obtain new results on bifurcation from many armed spirals.

The group orbit of the rotating spiral wave is three dimensional and the center subspace corresponding to Hopf bifurcation is two dimensional, thus leading to a five dimensional center bundle. With the construction of this bundle, we recover the action of the Euclidean group in Barkley's five dimensional model. The general equivariant vector field on the center bundle can then be analyzed.

It is our contention that center bundle techniques simplify the understanding of more complicated bifurcations from rotating waves. To illustrate this point, we discuss Hopf bifurcation from rotating waves recovering the results of Wulff [21] in the case of one-armed spirals and discovering new phenomena in the case of many armed spirals. We also discuss the codimension two Takens-Bogdanov bifurcation from one-armed spiral waves.

The center bundle reduction of Krupa [12] is formulated under the assumption that the total group of symmetries is compact and hence is not directly applicable to the problem of 
meandering spirals. However, it turns out that the main requirement is compactness of the isotropy subgroups of points on the critical group orbit. In the appendix, we prove, under certain hypotheses, that Krupa's theorems are valid even when the group of symmetries is not compact and acts on an infinite dimensional function space. In this theorem, it suffices that the isotropy subgroups are either discrete or compact, which is the case for spiral solutions since their isotropy subgroups are finite.

The hypotheses for the reduction described in the appendix are not satisfied in our particular context of Euclidean symmetry due to the nonsmoothness of the group action. Recent work of Sandstede et al. $[17,18]$ circumvents these hypotheses and enables the center bundle reduction for meandering spirals to be carried out rigorously.

\section{Center Bundles and Rotating Waves}

We begin by describing the relevant results in Krupa [12] on center bundles. Let $\Gamma$ be a compact Lie group acting orthogonally on $\mathbf{R}^{n}$, and let $f: \mathbf{R}^{n} \rightarrow \mathbf{R}^{n}$ be a $\Gamma$-equivariant vector field. A group orbit $X$ is a relative equilibrium if the flow of

$$
\dot{x}=f(x)
$$

leaves $X$ invariant. (Alternatively, $X$ is a relative equilibrium if $f$ is tangent to $X$ along $X$.) Note that rotating waves are relative equilibria as time evolution is the same as spatial rotation.

Suppose that the group orbit $X=\Gamma x_{0}$ is a relative equilibrium, and let $\Sigma \subset \Gamma$ be the isotropy subgroup of $x_{0}$. Let $T_{x_{0}} X$ be the tangent space to the group orbit at $x_{0}$, and note that $T_{x_{0}} X$ is $\Sigma$-invariant. Write

$$
\mathbf{R}^{n}=T_{x_{0}} X \oplus N_{x_{0}}
$$

where $N_{x_{0}}$ is the orthogonal complement to $T_{x_{0}} X$. We can form the normal bundle $N(X)$ over $X$ by attaching $\gamma N_{x_{0}}$ to the point $\gamma x_{0}$; since $N_{x_{0}}$ is $\Sigma$-invariant this construction makes sense and the bundle is $\Gamma$-invariant. This bundle gives a $\Gamma$-invariant tubular neighborhood of $X$ in $\mathbf{R}^{n}$.

Krupa then shows (see also Vanderbauwhede et al. [19]) that $f$ may be written as

$$
f=f_{N}+f_{T}
$$

on a neighborhood of $X$ where

- $f_{N}$ and $f_{T}$ are $\Gamma$-equivariant vector fields, 
- $f_{N}$ preserves normal fibers $\gamma N_{x_{0}}$, and

- $f_{T}$ is tangent to group orbits, that is, $f_{T}(x) \in T_{x} \Gamma x$.

It follows that $g=f_{N} \mid N_{x_{0}}$ is a $\Sigma$-equivariant vector field. Moreover, $g$ is 'generic' in the sense that any $\Sigma$-equivariant vector field $g: N_{x_{0}} \rightarrow N_{x_{0}}$ extends, in a neighborhood of $X$, to a $\Gamma$-equivariant vector field $f: \mathbf{R}^{n} \rightarrow \mathbf{R}^{n}$.

If $X$ is a relative equilibrium, then $g\left(x_{0}\right)=0$. The relative equilibrium $X$ is a critical group orbit if $(d g)_{x_{0}}$ has eigenvalues on the imaginary axis. (We note that $(d g)_{x_{0}}$ is computable, see Proposition A.3 in the appendix.) Let $V_{x_{0}}$ be the center subspace of $(d g)_{x_{0}}$. We call $V=\cup\left\{\gamma V_{x_{0}}\right\}$ the center bundle.

Next, Krupa shows that every solution $x(t)$ to the differential equation (2.1) near $X$ can be written as

$$
x(t)=\gamma(t) y(t),
$$

where $y(t)$ is a solution to the normal vector field equation $\dot{y}=g(y)$ and $\gamma(t) \in \Gamma$ is a smooth curve.

Suppose that $W_{x_{0}} \subset N_{x_{0}}$ is a $\Sigma$-invariant center manifold for $g$. Based on (2.2), Krupa observes that $W=\cup\left\{\gamma W_{x_{0}}\right\}$ is a $\Gamma$-invariant flow-invariant center manifold for $f$ in a neighborhood of the critical group orbit. In particular, if the noncritical eigenvalues of $(d g)_{x_{0}}$ all have negative real parts, then $W$ is attracting for the dynamics of $f$. As is usually the case with center manifolds, we can project $f \mid W$ onto the center bundle $V$. Thus, we can understand bifurcations from critical group orbits by studying bifurcations of the normal vector field $g$ from equilibria.

To apply the center bundle reduction to the problem of meandering spirals, it is necessary to generalize Krupa's results from compact groups to noncompact groups and from finite dimensions to infinite dimensions. We carry out this generalization, under certain natural hypotheses, in the appendix. We note however that there are additional technical problems coming from the action of the Euclidean group and it is necessary to appeal to the recent results of $[17,18]$. In the remainder of the main body of the paper, we show how these ideas can be formally applied to bifurcations from spiral waves in Euclidean equivariant systems.

\section{Group Action on the Center Bundle}

\section{Trivialization of center bundles}

We continue to use the notation from Section 2. In particular, $x_{0}$ is a point with isotropy $\Sigma$ and $X=\Gamma x_{0}$ is a critical relative equilibrium for the $\Gamma$-equivariant vector field $f$ on $N(X)$. 
Recall that the action of $\Gamma$ on points $(x, v) \in N(X)$ is given by $\gamma(x, v)=(\gamma x, \gamma v)$.

Define the $\Sigma$-equivariant vector field $g: N_{x_{0}} \rightarrow N_{x_{0}}$ as in Section 2 and let $V_{0}=V_{x_{0}}$ denote the $\Sigma$-invariant center subspace for $g$ with corresponding $\Gamma$-invariant center bundle $V=\cup\left\{\gamma V_{0}\right\}$. Although $N(X)$ is a trivial bundle, the subbundle $V$ is not necessarily trivial (see Remark 3.4). We now give a sufficient condition for $V$ to be a trivial bundle.

Lemma 3.1 Suppose that the action $\rho$ of $\Sigma$ on $V_{0}$ extends to an action $\rho$ of $\Gamma$ on $V_{0}$. Then there is a trivialization

$$
V \cong X \times V_{0}
$$

The action of $\Gamma$ on $V$ is given by

$$
\gamma(x, v)=\left(\gamma x, \rho_{\gamma} v\right)
$$

where $\gamma \in \Gamma, x \in X, v \in V_{0}$.

Proof Let $(x, w) \in V$, so $x \in X$ and $w \in V_{x}$ where $V_{x}$ is the fiber over $x$. Write $x=\delta x_{0}$ where $\delta \in \Gamma$ and observe that $V_{x}=\delta V_{0}$. Hence $\delta^{-1} w \in V_{0}$. Define the trivialization $h: V \rightarrow X \times V_{0}$ by

$$
h(x, w)=\left(x, \rho_{\delta}\left(\delta^{-1} w\right)\right) .
$$

To show that $h$ is well-defined, suppose that $x=\delta_{1} x_{0}=\delta_{2} x_{0}$ where $\delta_{1}, \delta_{2} \in \Gamma$. Then $\delta_{2}^{-1} \delta_{1}=\sigma \in \Sigma$. The assumption on the action $\rho$ ensures that $\rho_{\sigma} v=\sigma v$ for all $v \in V_{0}$. We compute that

$$
\rho_{\delta_{2}}\left(\delta_{2}^{-1} w\right)=\rho_{\delta_{2}} \rho_{\sigma}\left(\sigma^{-1} \delta_{2}^{-1} w\right)=\rho_{\delta_{2}} \rho_{\delta_{2}^{-1} \delta_{1}}\left(\delta_{1}^{-1} \delta_{2} \delta_{2}^{-1} w\right)=\rho_{\delta_{1}}\left(\delta_{1}^{-1} w\right)
$$

hence $h$ is well-defined.

Next, we verify the action of $\Gamma$ on $X \times V_{0}$. In other words, we show that $h$ is $\Gamma$-equivariant with respect to the actions on $V$ and $X \times V_{0}$. Let $(x, w) \in V$ as at the beginning of the proof. Then

$$
\begin{aligned}
h(\gamma(x, w)) & =h(\gamma x, \gamma w)=\left(\gamma x, \rho_{\gamma \delta}\left((\gamma \delta)^{-1} \gamma w\right)\right) \\
& =\left(\gamma x, \rho_{\gamma} \rho_{\delta}\left(\delta^{-1} w\right)\right)=\gamma\left(x, \rho_{\delta}\left(\delta^{-1} w\right)\right)=\gamma h(x, w),
\end{aligned}
$$

as required.

The next corollary includes the case $\Sigma=\mathbf{1}$ (one-armed spirals).

Corollary 3.2 Suppose that $\Sigma$ acts trivially on $V_{0}$. Then $V \cong X \times V_{0}$ is a trivial bundle and $\Gamma$ acts as $(x, v) \rightarrow(\gamma x, v)$. 
Next, we prove a general result about Hopf bifurcation from a relative equilibrium when $\Gamma=\mathbf{S O}(2)$.

Proposition 3.3 Suppose that $\Gamma=\mathbf{S O}(2)$ and that $X=\Gamma x_{0}$ is a relative equilibrium. If $X$ undergoes a Hopf bifurcation, then generically $\operatorname{dim} V_{0}=2$ and the corresponding center bundle $V=X \times V_{0}$ is a trivial bundle.

Proof If $\Sigma=\mathbf{S O}(2)$, then $X$ is a point and the result is immediate. Hence, we may suppose that $\Sigma=\mathbf{Z}_{\ell}, \ell \geq 1$, with generator $\sigma=R_{2 \pi / \ell} \in \mathbf{S O}(2)$. Each irreducible representation of $\Sigma$ is one dimensional (and absolutely irreducible) or two dimensional (and nonabsolutely irreducible). It follows from general theory [8] that generically either $V_{0}$ is nonabsolutely irreducible or $V_{0}$ is the sum of two isomorphic absolutely irreducible representations. Either way, $\operatorname{dim} V_{0}=2$. Moreover, $\sigma$ acts as an orientation-preserving transformation on $V_{0}$ : $\sigma z=e^{2 \pi i m / \ell} z$ for some $m=0, \ldots,[\ell / 2]$. To apply Lemma 3.1, take $\rho_{\theta} z=e^{i m \theta} z, \theta \in \mathbf{S O}(2)$.

Remark 3.4 (a) We give a simple example of a bifurcation from a relative equilibrium for which the center bundle is not a trivial bundle. Take $\Gamma=\mathbf{S O}(2), \Sigma=\mathbf{Z}_{2}, V_{0} \cong \mathbf{R}$ and suppose that $\Sigma$ acts nontrivially on $V_{0}$. (In other words, we consider a symmetry-breaking steady-state bifurcation in the normal vector field.) Then the center bundle $V$ is a Möbius band.

(b) By results of Fiedler et al. [6], the analysis that follows does not depend crucially on whether or not $V$ is a trivial bundle.

\section{The center bundle for spirals}

Now suppose that $\Gamma=\mathbf{S E}(2)$, the special Euclidean group consisting of rotations and translations. We suppose that $X=\mathbf{S E}(2) x_{0}$ is a relative equilibrium where $x_{0}$ is an $\ell$-armed spiral. In other words the isotropy subgroup $\Sigma \cong \mathbf{Z}_{\ell}$. As a manifold $\mathbf{S E}(2)$ is diffeomorphic to $\mathbf{R}^{2} \times \mathbf{S}^{1}$. The assumptions on the symmetry of $x_{0}$ imply that

$$
X=\mathbf{S E}(2) x_{0} \cong \mathbf{S E}(2) / \mathbf{Z}_{\ell} \cong \mathbf{R}^{2} \times\left(\mathbf{S}^{1} / \mathbf{Z}_{\ell}\right) \cong \mathbf{C} \times \mathbf{S}^{1},
$$

that is, $X$ is a cylinder with coordinates $(p, \varphi)$.

Lemma 3.5 The action of $(x, \theta) \in \mathbf{S E}(2)$ on $(p, \varphi) \in X$ is:

$$
(x, \theta)(p, \varphi)=\left(e^{i \theta} p+x, \varphi+\ell \theta\right) .
$$


Proof To verify (3.1), note that the action of $\mathbf{S E}(2)$ on $X$ is just induced by the action of group multiplication in $\mathbf{S E}(2)$. Group multiplication in $\mathbf{S E}(2)$ is most easily understood through the action of $\mathbf{S E}(2)$ on $\mathbf{R}^{2}=\mathbf{C}$. Let $w \in \mathbf{C}$; then

$$
(x, \theta) w=e^{i \theta} w+x .
$$

It follows that

$$
(x, \theta)(y, \psi) w=(x, \theta)\left(e^{i \psi} w+y\right)=e^{i \theta}\left(e^{i \psi} w+y\right)+x=e^{i(\theta+\psi)} w+\left(e^{i \theta} y+x\right) .
$$

Hence, the group multiplication on $\mathbf{S E}(2)$ induced by its action on $\mathbf{C}$ is

$$
(x, \theta)(y, \psi)=\left(e^{i \theta} y+x, \psi+\theta\right)
$$

Substituting $(p, \varphi)$ for $(y, \psi)$ gives the action of $\mathbf{S E}(2)$ on $X$ when $\ell=1$. For general $\ell$, the angle $\theta$ acts on $\mathbf{C}$ as in the $\ell=1$ case, but $\theta$ acts on $\mathbf{S}^{1} / \mathbf{Z}_{\ell}$ as an $\ell$-fold covering. That is, we must add $\ell \theta$ to $\varphi$.

Lemma 3.6 Hopf bifurcation from an $\ell$-armed spiral reduces generically to Hopf bifurcation of a five dimensional vector field on a trivial center bundle

$$
V=X \times V_{0}
$$

that is equivariant under the action:

$$
\begin{aligned}
& T_{x}(p, \varphi, q)=(p+x, \varphi, q) \\
& R_{\theta}(p, \varphi, q)=\left(e^{i \theta} p, \varphi+\ell \theta, e^{m i \theta} q\right)
\end{aligned}
$$

where $0 \leq m \leq[\ell / 2]$.

Proof Whether or not $V$ is a trivial bundle is independent of the $\mathbf{C}$ factor in $X$. Hence, it follows from Proposition 3.3 that $V$ is a trivial bundle. The action of $\mathbf{S E}(2)$ on the $X$ coordinates follows from (3.1). The action on the $V$ coordinates follows from Lemma 3.1 and the proof of Proposition 3.3. 


\section{Meandering and Resonant Growth of a One-Armed Spiral}

In this section, we analyze Hopf bifurcation from a one-armed spiral. In Subsection 4.1, we write down the general equivariant vector field on the center bundle. In Subsection 4.2, we solve these equations and obtain the conditions for resonant growth. Finally, in Subsection 4.3, we interpret these results in the context of Hopf bifurcation in a partial differential equation, recovering the results of Barkley and Wulff on the meandering and resonant growth of spirals.

\subsection{Equivariant vector fields on the center bundle}

For a one-armed spiral, we have $\ell=1, m=0$ in Lemma 3.6.

Lemma 4.1 Let $F$ be a system of differential equations on the center bundle $V$ that is $\mathrm{SE}(2)$-equivariant with respect to the $\ell=1, m=0$ action. Then $F$ has the form

$$
\begin{aligned}
& \text { (a) } \dot{p}=e^{i \varphi} f(q) \\
& \text { (b) } \dot{\varphi}=F^{\varphi}(q) \\
& \text { (c) } \dot{q}=F^{q}(q) .
\end{aligned}
$$

Proof Symmetry invariance of a system of differential equations means that solutions are transformed to solutions by that symmetry. Thus, translation invariance implies that if

$$
z(t)=(p(t), \varphi(t), q(t))
$$

is a solution to (4.1), then so is

$$
y(t)=(p(t)+x, \varphi(t), q(t))
$$

for any $x \in \mathbf{C}$. Since $\dot{z}(t)=\dot{y}(t)$, it follows that

$$
F(z(t))=F(y(t))
$$

for all solutions $z(t)$. In particular,

$$
F(p+x, \varphi, q)=F(p, \varphi, q),
$$


for all $x$. Hence $F$ is independent of $p$ and the differential equations have the form

$$
\begin{aligned}
\dot{p} & =F^{p}(\varphi, q) \\
\dot{\varphi} & =F^{\varphi}(\varphi, q) \\
\dot{q} & =F^{q}(\varphi, q) .
\end{aligned}
$$

Similarly, the rotational invariance of (4.2) implies that

$$
\begin{aligned}
& F^{p}(\varphi+\theta, q)=e^{i \theta} F^{p}(\varphi, q) \\
& F^{\varphi}(\varphi+\theta, q)=F^{\varphi}(\varphi, q), \\
& F^{q}(\varphi+\theta, q)=F^{q}(\varphi, q) .
\end{aligned}
$$

Thus, $F^{\varphi}$ and $F^{q}$ are independent of $\varphi$, which verifies $(4.1)(\mathrm{b}, \mathrm{c})$.

To complete this proof we must verify $(4.1)(\mathrm{a})$. Define

$$
H(\varphi, q)=e^{-i \varphi} F^{p}(\varphi, q)
$$

and note that

$$
H(\varphi+\theta, q)=e^{-i(\varphi+\theta)} F^{p}(\varphi+\theta, q)=e^{-i \varphi} e^{-i \theta} e^{i \theta} F^{p}(\varphi, q)=H(\varphi, q) .
$$

It follows that $H(\varphi, q)=f(q)$ is independent of $\varphi$ and that $(4.1)($ a) is valid.

\subsection{Periodic Solutions and Resonant Growth}

Suppose that $q(t)$ is a $2 \pi / \omega_{2}$ periodic solution to

$$
\dot{q}=F^{q}(q)
$$

in the center bundle equations (4.1). We define $\omega_{1}=F^{\varphi}(q(0))$. (These frequencies are related to, but not identical to, the frequencies $\omega_{1}$ and $\omega_{2}$ that appear in the introduction, see equation (4.7).)

We can solve

$$
\dot{\varphi}=F^{\varphi}(q(t))
$$

for $\varphi(t)$ and

$$
\dot{p}=e^{i \varphi(t)} f(q(t))
$$

for $p(t)$ to obtain a solution $(p(t), \varphi(t), q(t))$ to $(4.1)$. In the next theorem we recover the resonance conditions of Barkley and Wulff for these solutions. 
Theorem 4.2 Let $(p(t), \varphi(t), q(t))$ be a solution to (4.1). Then

$$
\varphi(t)=\omega_{1} t+\tilde{\varphi}(t)
$$

where $\tilde{\varphi}(t)$ is $2 \pi / \omega_{2}$ periodic. If

$$
\omega_{1}+k \omega_{2}=0
$$

for some integer $k$, then generically $p(t)$ undergoes unbounded resonant growth.

Proof The function $F^{\varphi}(q(t))$ is $2 \pi / \omega_{2}$ periodic since $q(t)$ is. Therefore, we can write $F^{\varphi}(q(t))$ as a Fourier series in $t$ obtaining

$$
\dot{\varphi}=\sum_{n=-\infty}^{\infty} B_{n} e^{i n \omega_{2} t}
$$

where $B_{n} \in \mathbf{C}$ and $B_{-n}=\overline{B_{n}}$. Every term except $n=0$ in the Fourier series yields a periodic function on integration and hence $\varphi(t)$ has the form in (4.3) where $\omega_{1}=B_{0}=F^{\varphi}(q(0))$.

Next, consider the differential equation

$$
\dot{p}=e^{i \varphi(t)} f(q(t))=e^{i \omega_{1} t} H(t)
$$

where $H(t)$ is smooth and $2 \pi / \omega_{2}$ periodic. We may write $H(t)$ as the uniformly convergent Fourier series

$$
H(t)=\sum_{n=-\infty}^{\infty} D_{n} e^{i n \omega_{2} t},
$$

where $D_{n} \in \mathbf{C}$.

Suppose that $\omega_{1}+k \omega_{2}$ is close to zero for some nonzero integer $k$. Then integration of (4.4) yields

$$
p(t)= \begin{cases}D_{k} t+\mathcal{P}(t) e^{i \omega_{1} t} & \omega_{1}+k \omega_{2}=0 \\ \frac{D_{k}}{i\left(\omega_{1}+k \omega_{2}\right)} e^{i\left(\omega_{1}+k \omega_{2}\right) t}+\mathcal{P}(t) e^{i \omega_{1} t} & \omega_{1}+k \omega_{2} \neq 0\end{cases}
$$

where $\mathcal{P}(t)$ is a smooth bounded $2 \pi / \omega_{2}$ periodic function. Generically, $D_{k} \neq 0$. Hence, by varying $\omega_{2}$ so that $\omega_{1}+k \omega_{2}$ goes through zero, the first summand in $p(t)$ undergoes unbounded resonant growth, while $\mathcal{P}(t)$ remains uniformly bounded for these values of $\omega_{2}$. 


\subsection{Hopf Bifurcation from a One-Armed Spiral}

Let $\mathcal{H}$ be a space of functions with domain $\mathbf{R}^{2}$ on which the Euclidean group $\mathbf{E}(2)$ acts as

$$
\gamma u(z)=u\left(\gamma^{-1} z\right),
$$

where $u \in \mathcal{H}$ and $\gamma \in \mathbf{E}(2)$. Consider a partial differential equation

$$
u_{t}=\mathcal{F}(u, \lambda)
$$

where $\mathcal{F}: \mathcal{H} \mapsto \mathcal{H}$ is $\mathbf{E}(2)$-equivariant and $\lambda$ is a real bifurcation parameter. Let $R_{\theta}$ denote rotation of the plane through angle $\theta$. Suppose that

$$
u(t)=R_{\omega_{1} t} x_{0}
$$

is a rotating wave solution to (4.5) with period $2 \pi / \omega_{1}$.

Let $X=\mathbf{S E}(2) x_{0}$ be the connected component of the group orbit of $u(t)$ in phase space under the action of $\mathbf{E}(2)$. As noted by Rand [15], Renardy [16] and others, it is possible to study bifurcation from rotating waves by transferring the problem to the rotating frame. Substituting (4.6) into (4.5) yields that $x_{0}$ is an equilibrium for the equation

$$
u_{t}=\tilde{\mathcal{F}}(u, \lambda)=\mathcal{F}(u, \lambda)-\omega_{1} \xi u,
$$

where

$$
\xi u=\left.\frac{d}{d t} R_{t} u\right|_{t=0} .
$$

The operator $(d \tilde{\mathcal{F}})_{x_{0}, \lambda}$ has three eigenvalues on the imaginary axis corresponding to the continuous group orbit $\mathbf{S E}(2)$. Barkley [2] showed numerically that the rotating wave $u(t)$ could undergo a Hopf bifurcation as an additional simple pair of eigenvalues cross the imaginary axis. We suppose that this bifurcation occurs at $\lambda=0$. Let $V_{0} \cong \mathbf{C}$ be the corresponding center subspace.

Theorem $4.3[17,18]$ There exists a reduction of (4.5) to the center bundle $V=X \times V_{0}$. The reduced equations have the form

$$
\dot{y}=F(y, \lambda),
$$

where $y=(p, \varphi, q) \in V$ and $F$ has the form in equations (4.1). 
It follows from the reduction procedure that

$$
F^{\varphi}(0,0)=\omega_{1}, \quad F^{q}(0,0)=0, \quad \text { and } \quad f(0,0)=0 .
$$

Note that in equation (4.1), the original rotating wave solution corresponds to the equilibrium $q=0$. Also, the critical eigenvalues generically cross the imaginary axis transversely on variation of $\lambda$. Consequently, the vector field $F^{q}(q, \lambda)$ on $V_{0}$ satisfies

$$
d_{q} F^{q}(0,0)=i \omega_{2} \quad \text { and } \quad \operatorname{Re} d_{q} F_{\lambda}^{q}(0,0) \neq 0 .
$$

Thus, there is a Hopf bifurcation in the $\dot{q}$ equation of (4.1) to an approximately $2 \pi / \omega_{2}$ periodic solution $q(t)$. We suppose that the Hopf bifurcation is supercritical.

The amplitude and frequency of the periodic solution $q(t, \lambda)$ vary as functions of $\lambda$. To leading order, the amplitude varies as $a \sqrt{\lambda}$ and the frequency varies as $\omega_{2}+b \lambda$ where $a$ and $b$ are real coefficients. We set $\omega_{1}(\lambda)=F^{\varphi}(q(0, \lambda))$ and define $\omega_{2}(\lambda)$ to be the frequency of $q(t, \lambda)$. Thus

$$
\omega_{1}(\lambda)=\omega_{1}+\mathcal{O}(\sqrt{\lambda}), \quad \omega_{2}(\lambda)=\omega_{2}+\mathcal{O}(\lambda) .
$$

Note that $\omega_{j}(0)$ coincides with $\omega_{j}$ as defined in this subsection and in the introduction for each $j=1,2$. On the other hand, the $\omega_{j}$ 's in Subsection 4.2 correspond to $\omega_{j}(\lambda)$ evaluated at a specific value of $\lambda$.

It follows from Theorem 4.2 that linear meandering occurs at $\lambda=\lambda_{0}$ if

$$
\omega_{1}\left(\lambda_{0}\right)+k \omega_{2}\left(\lambda_{0}\right)=0
$$

for some integer $k$. We call this resonance a $k$-resonance. In particular, resonant growth occurs when $\omega_{1}(0)+k \omega_{2}(0)$ is close to zero for some integer $k$. We can expect unbounded growth in $p(t)$ as $\lambda$ approaches the resonance point, and linear drifting in $p(t)$ at the resonance point. However, by inspection of pictures, only when $k= \pm 1$ or $k= \pm 2$ do the concepts of petality and changes in petality appear to be relevant.

\section{Visualization of Hopf Bifurcation from a One-Armed Spiral}

To illustrate resonance and petality issues, we have plotted results of numerical integration of the equations (4.8) in Figure 3 for six values of $\lambda$.

$$
\begin{aligned}
& \dot{p}=e^{i \varphi}(0.2-0.6 i) q \\
& \dot{\varphi}=1 \\
& \dot{q}=(\lambda-0.95 i) q-(1-0.1 i) q|q|^{2}
\end{aligned}
$$


Each figure is a plot of $\left(f_{1}, f_{2}\right)$ where

$$
f_{1}=\cos \varphi(t)+\operatorname{Re} p(t) \quad \text { and } \quad f_{2}=\sin \varphi(t)+\operatorname{Im} p(t) .
$$

These coordinates approximate the movements of the spiral tip in the lab frame. The $\dot{q}$ equation in (4.8) undergoes a supercritical Hopf bifurcation at $\lambda=0$, and the frequency of the corresponding periodic solution is $\omega_{2}(\lambda)=-0.95-0.1 \lambda$. Since $\omega_{1}(\lambda) \equiv 1$, there is a resonance at $\lambda=0.5$.
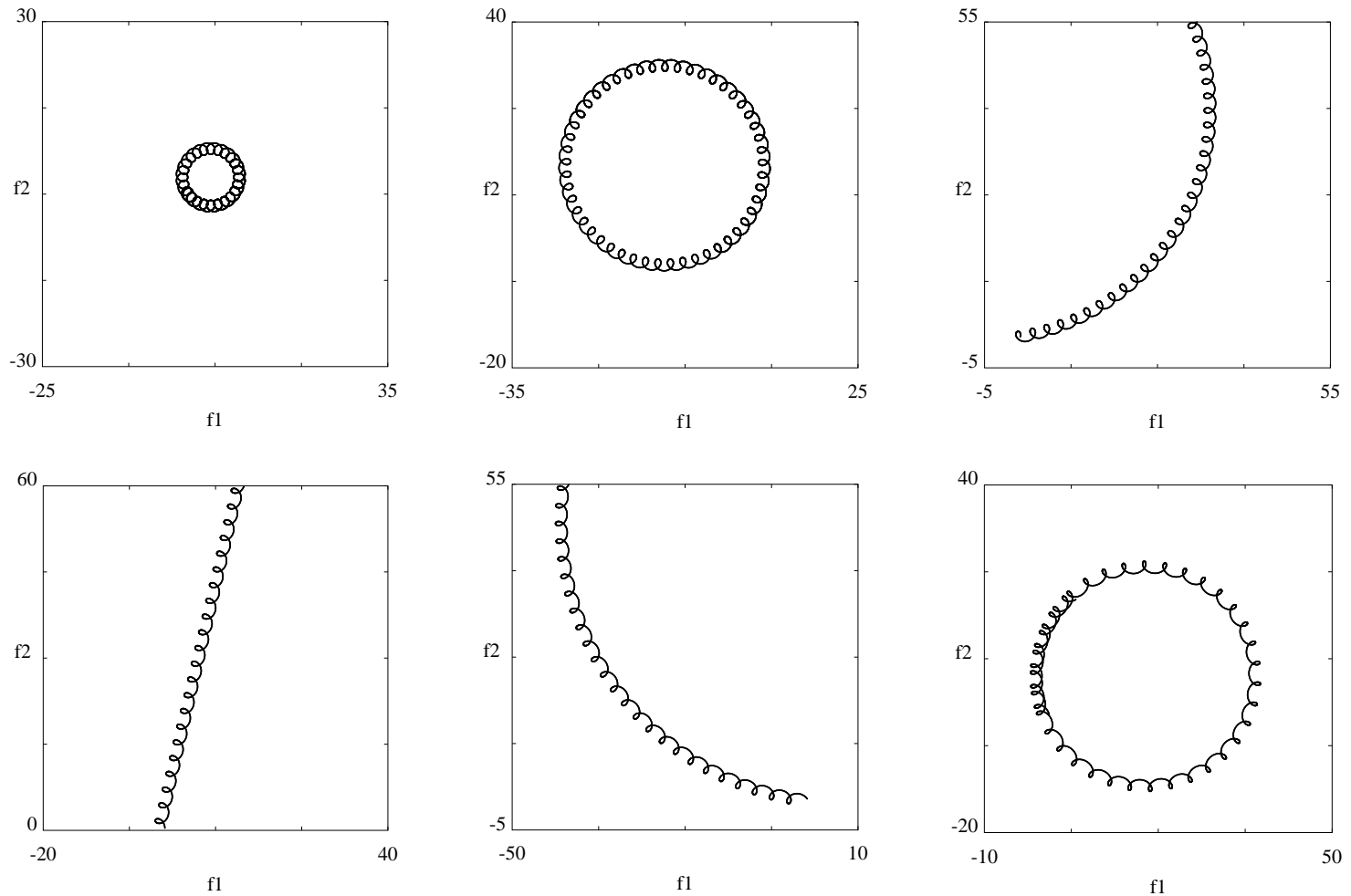

Figure 3: Numerical simulation of (4.8) for $\lambda=0.1,0.3,0.4,0.5,0.6$ and 0.8 .

As previously mentioned, in order to see well-defined petals in the simple epicycle motion described by (1.1), the amplitude of the second frequency must be larger than the amplitude 
of the primary frequency. That is, in order to see well-defined petals, we must have

$$
\frac{\left|z_{2}\right|}{\left|z_{1}\right|}>M
$$

for some real positive number $M$. Suppose that the secondary motion arises via a Hopf bifurcation and that $\left|z_{1}\right| \approx 1$. Then near a point of $k$-resonance, we have

$$
\frac{\left|z_{2}\right|}{\left|z_{1}\right|} \approx \frac{\sqrt{\lambda}\left|D_{k}\right|}{\omega_{1}\left(1+k \frac{\omega_{2}}{\omega_{1}}\right)}
$$

where $D_{k}$ is a Fourier coefficient and we have suppressed the dependence of $\omega_{1}, \omega_{2}$ on $\lambda$. Thus, if we define $\tau=\omega_{2} / \omega_{1}$, then (4.9) becomes

$$
\lambda>\frac{M^{2} \omega_{1}^{2}}{\left|D_{k}\right|^{2}}(1+k \tau)^{2} .
$$

This defines a sequence of "resonance tongues" in the $\tau \lambda$ plane in which changes of petality can be observed in addition to unbounded growth of the flower near the resonance. See Figure 4. Since $\lim _{k \rightarrow \infty}\left|D_{k}\right|=0$, the tongues eventually narrow as $k \rightarrow \infty$.

If the point $(\tau, \lambda)$ is in exactly one tongue (as is the point labeled A in Figure 4), then an epicycle motion with well-defined petals will be observed. A change in petality occurs as the resonance is crossed. If the point is in a tongue corresponding to a $k$ resonance, then each petal will be (approximately) traced out $k$ times before a new petal is formed. However, in the case where the point is in more than one tongue (as is point B in Figure 4), then the motion involves multiple harmonics and petality is an ill-defined concept for these points. However, we will still observe unbounded growth of the flower as the resonance is crossed.

\section{Hopf Bifurcation from $\ell$-Armed Spirals}

In this section we discuss Hopf bifurcation from $\ell$-armed spirals where $\ell>1$. Suppose that an $\ell$-armed spiral wave undergoes a simple Hopf bifurcation with frequency $\omega_{2}$. Let $V_{0}$ be the center subspace of the bifurcation in the normal directions to $X$ at $x_{0}$. The $\mathbf{S E}(2)$ action is given as in (3.2):

$$
(p, \varphi, q) \mapsto\left(e^{i \theta} p+x, \varphi+\ell \theta, e^{i m \theta} q\right)
$$

for some $m=0, \ldots,[\ell / 2]$. 


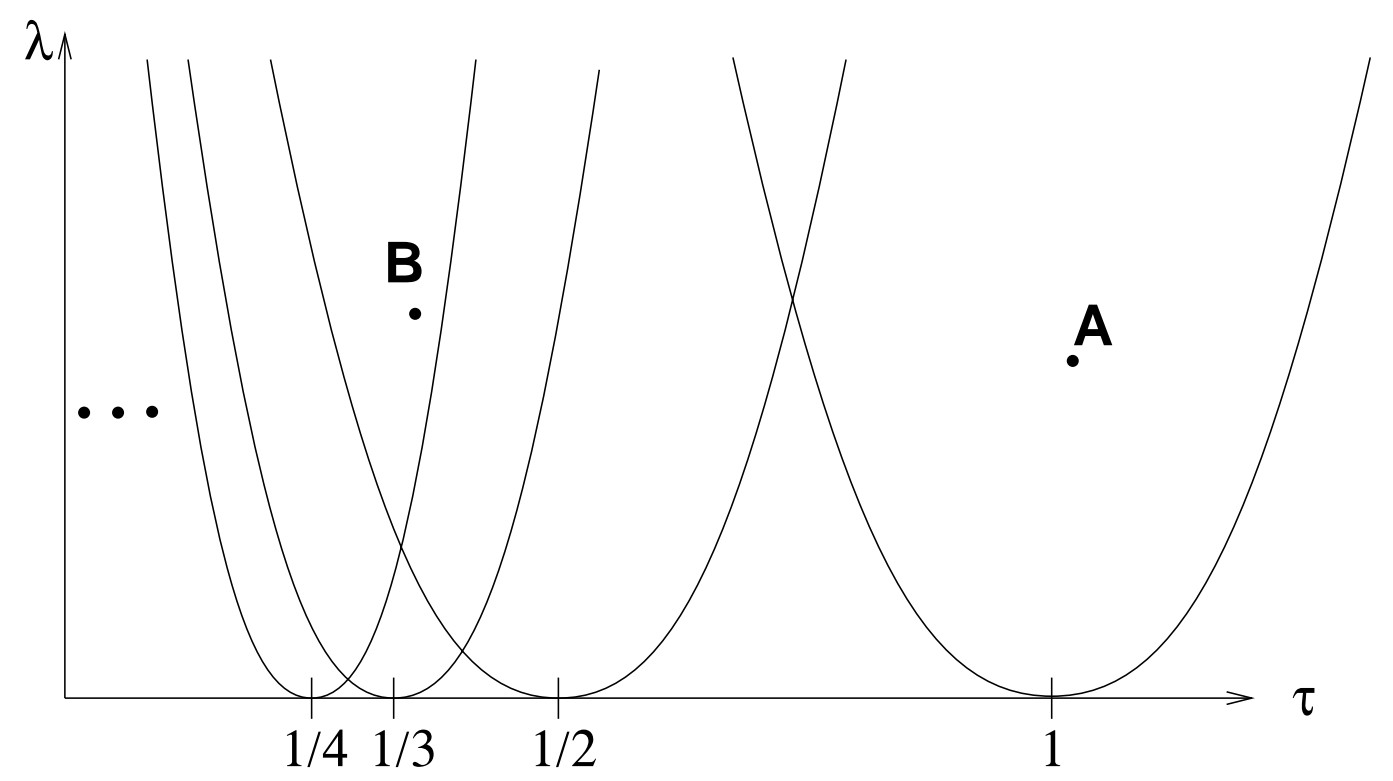

Figure 4: Resonance tongues described by (4.10).

The isotropy subgroup in $\mathbf{S E}(2)$ of the $\ell$-armed spiral is $\mathbf{Z}_{\ell}$. Note that the action of $\mathbf{Z}_{\ell}$ on $V_{0}$ is faithful (in other words, the kernel of the action is trivial) precisely when $\ell$ and $m$ are coprime. For example, if the eigenfunction associated with Hopf bifurcation from a two-armed spiral $(\ell=2)$ is invariant under rotation by $\pi$, then $m=0$, and $\ell$ and $m$ are not coprime.

For all $\ell$ and $m$, the solutions arising from Hopf bifurcation are quasiperiodic. However, there are differences in the resulting motions in physical space depending on whether or not $\ell$ and $m$ are coprime. In particular:

- When $\ell$ and $m$ are not coprime, the spiral tip does not meander and the codimension two bifurcation to resonant growth does not occur. What does occur is that (approximately) the spiral rigidly rotates at a rate that depends quasiperiodically on time.

- When $\ell$ and $m$ are coprime, Hopf bifurcation leads to meandering and to codimension two resonant growth. The points where resonance occurs depend on both $\ell$ and $m$.

In the remainder of this section, we give the precise statements and proofs of these results. 


\section{Hopf Bifurcations without Meandering}

First, we analyze the equations on the center bundle when $\ell$ and $m$ are not coprime. We prove:

Theorem 5.1 Let $F$ be an $\mathbf{S E}(2)$-equivariant system of differential equations on the center bundle $V$ corresponding to an $\ell$-armed spiral where $\ell>1$. If $\ell$ and $m$ are not coprime, then $F^{p}=0$ and $p(t)$ is constant. In particular, there is no resonant growth.

Moreover, the spiral in the physical plane does not meander. Rather, the motion is a rigid rotation by $R_{\varphi(t)}$, where $\varphi(t)$ is quasiperiodic in $t$.

Proof As in the proof of Lemma 4.1, the action of translations on $V$ implies that $F$ is independent of $p$. The main difference comes in the equation

$$
\dot{p}=F^{p}(\varphi, q) \text {. }
$$

Here the action of rotations implies that

$$
e^{i \theta} F^{p}(\varphi, q)=F^{p}\left(\varphi+\ell \theta, e^{m i \theta} q\right)
$$

for all $\theta$. Since $\ell$ and $m$ are not coprime there is an integer $j \geq 2$ such that $\ell=j \ell^{\prime}$ and $m=j m^{\prime}$. Setting $\theta=2 \pi / j$ in (5.1) yields

$$
e^{2 \pi i / j} F^{p}(\varphi, q)=F^{p}\left(\varphi+2 \ell^{\prime} \pi, e^{2 m^{\prime} \pi i} q\right)=F^{p}(\varphi, q) .
$$

Since $j>1$ it follows that $F^{p}=0$. In particular, $p(t)$ is constant and there is no resonant growth and no meandering.

The remaining components of the system on the five dimensional center bundle have the form

$$
\begin{aligned}
\dot{\varphi} & =F^{\varphi}(\varphi, q) \\
\dot{q} & =F^{q}(\varphi, q) .
\end{aligned}
$$

This can be viewed as an $\mathbf{S O}(2)$-equivariant system on the three dimensional center bundle $\mathbf{S O}(2) x_{0} \times V_{0}$. In these equations we have a Hopf bifurcation in the normal $(q)$ directions leading to a periodic solution; and drift along the $\mathbf{S O}(2)$ group orbit leading to a quasiperiodic $\varphi(t)$ — just as in the case of one-armed spirals.

The interpretation of $\varphi(t)$ in physical space is that the plane is rigidly rotating by $R_{\varphi(t)}$. (In the case of one-armed spirals, the plane also translates since $p(t)$ is not constant.) In the corresponding quasiperiodically varying rotating frame, the spiral is approximately stationary. In fact, this spiral also varies quasiperiodically (as a function of $q(t)$ ), but close to the point of Hopf bifurcation, this fluctuation is negligible. 
Remark 5.2 There is an alternative abstract explanation of the failure of the spiral tip to meander or undergo resonant growth when $\ell$ and $m$ are not coprime, based on ideas in $[7,12]$. Since the kernel of the action of $\mathbf{Z}_{\ell}$ on $V_{0}$ is nontrivial, the bifurcating periodic solutions are fixed pointwise by the kernel $\mathbf{Z}_{k}$ of the action. (Here $k$ divides $\ell$ and $k>1$ by assumption.) Hence the bifurcation takes place in the fixed-point subspace $\operatorname{Fix}\left(\mathbf{Z}_{k}\right)$. As usual, this is a flow-invariant subspace for the underlying $\mathbf{S E}(2)$-equivariant vector field. Moreover, the largest subgroup of $\mathbf{S E}(2)$ that preserves $\operatorname{Fix}\left(\mathbf{Z}_{k}\right)$ is the normalizer $\mathbf{S O}(2)$ of $\mathbf{Z}_{k}$. Hence the flow on $\operatorname{Fix}\left(\mathbf{Z}_{k}\right)$ is an $\mathbf{S O}(2)$-equivariant flow and all drifts take place inside $\mathrm{SO}(2)$. In particular, the translation coordinate on the center bundle remains constant.

\section{Hopf Bifurcations with Meandering}

Now assume that $\ell$ and $m$ are coprime. As usual, let $\omega_{1}$ denote the frequency of the basic $\ell$-armed spiral solution and let $\omega_{2}$ be the frequency coming from Hopf bifurcation. Our main result is that the conditions for resonant growth depend on both $\ell$ and $m$ in the following manner.

Theorem 5.3 Assume that $\ell$ and $m$ are coprime and let $m_{0}$ be the smallest positive integer such that

$$
m m_{0} \equiv 1 \quad(\bmod \ell) .
$$

Then codimension two resonant growth occurs when

$$
\omega_{2} \sim \frac{1}{\ell}\left(m-\frac{1}{j \ell+m_{0}}\right) \omega_{1},
$$

where $j$ is an integer.

For example, when $\ell=2$ and $m=1$, the resonance condition becomes

$$
\omega_{2} \sim \frac{j}{2 j+1} \omega_{1},
$$

where $j$ is an integer.

The proof of Theorem 5.3 is organized as follows.

- First, we calculate the equivariant vector field on the center bundle. To make the analysis of this vector field tractable, we consider a pull-back to a vector field on the group. This step is a special case of the more general approach of Fiedler et al. [6]. 
Indeed, we wish to thank Bernold Fiedler for showing us that certain computational difficulties can be circumvented by pulling the differential equation from the center bundle over the group orbit back to a differential equation on a bundle over the group itself

- Second, we compute necessary and sufficient conditions for resonant growth in the pull-back equations.

- Finally, we reinterpret these results for the original vector field on the center bundle.

The action of $\mathbf{S E}(2)$ on the center bundle $V$ is given in Lemma 3.6. Unlike the $\ell=1$ case, the $\dot{\varphi}$ and $\dot{q}$ equations depend on $\varphi$. In fact, the general $\mathbf{S E}(2)$-equivariant system of differential equations on $V$ when $\ell>1$ has the form

$$
\begin{aligned}
\dot{p} & =F^{p}(\varphi, q) \\
\dot{\varphi} & =F^{\varphi}(\varphi, q) \\
\dot{q} & =F^{q}(\varphi, q),
\end{aligned}
$$

where

$$
\begin{aligned}
& F^{p}\left(\varphi+\ell \theta, e^{m i \theta} q\right)=e^{i \theta} F^{p}(\varphi, q) \\
& F^{\varphi}\left(\varphi+\ell \theta, e^{m i \theta} q\right)=F^{\varphi}(\varphi, q) \\
& F^{q}\left(\varphi+\ell \theta, e^{m i \theta} q\right)=e^{m i \theta} F^{q}(\varphi, q) .
\end{aligned}
$$

The techniques which were used in the $\ell=1$ case to study resonances will not work for equations (5.3) because of the dependence of the equations on $\varphi$. To circumvent this difficulty, define the local diffeomorphism

$$
\begin{gathered}
\rho: \mathbf{S E}(2) \times V_{0} \longrightarrow V \\
\rho(y, \psi, v)=\left(y, \ell \psi, e^{m i \psi} v\right),
\end{gathered}
$$

where $0 \leq \psi<2 \pi$. Define an action of $\mathbf{S E}(2)$ on the bundle $\mathbf{S E}(2) \times V_{0}$ by:

$$
(x, \theta)(y, \psi, v)=\left(e^{i \theta} y+x, \psi+\theta, v\right), \quad(x, \theta) \in \mathbf{S E}(2) .
$$

With this action, $\rho$ is $\mathbf{S E}(2)$ equivariant. To verify this point, calculate

$$
\rho((x, \theta)(y, \psi, v))=\rho\left(e^{i \theta} y+x, \psi+\theta, v\right)=\left(e^{i \theta} y+x, \ell(\psi+\theta), e^{m i(\psi+\theta)} v\right),
$$


and, using (3.2), calculate

$$
(x, \theta) \rho(y, \psi, v)=(x, \theta)\left(y, \ell \psi, e^{m i \psi} v\right)=\left(e^{i \theta} y+x, \ell \psi+\ell \theta, e^{m i \theta} e^{m i \psi} v\right) .
$$

Using $\rho$, the differential equation (5.3) pulls back to a differential equation on $\mathrm{SE}(2) \times V_{0}$ that is equivariant under the action (5.5) of $\mathbf{S E}(2)$. Since this action is identical to the $\ell=1$, $m=0$ action encountered in Section 4, it follows from Lemma 4.1 that the pull-back has the form

$$
\begin{aligned}
\dot{y} & =e^{i \psi} g(v) \\
\dot{\psi} & =G^{\psi}(v) \\
\dot{v} & =G^{v}(v) .
\end{aligned}
$$

Proposition 5.4 The pull-back equations on the group are related to the original equations on the group orbit as follows.

$$
\begin{aligned}
g(v) & =F^{p}(0, v) \\
G^{\psi}(v) & =\frac{1}{\ell} F^{\varphi}(0, v) \\
G^{v}(v) & =F^{q}(0, v)-\frac{m i}{\ell} v F^{\varphi}(0, v) .
\end{aligned}
$$

Proof The function $(y(t), \psi(t), v(t))$ is a solution to the pull-back differential equation (5.6) if and only if $\rho(y(t), \psi(t), v(t))=\left(y(t), \ell \psi(t), e^{m i \psi(t)} v(t)\right)$ is a solution to (5.3). Thus, differentiation leads to

$$
\begin{gathered}
\dot{y}=F^{p}\left(\ell \psi, e^{m i \psi} v\right) \\
\ell \dot{\psi}=F^{\varphi}\left(\ell \psi, e^{m i \psi} v\right) \\
m i e^{m i \psi} v \dot{\psi}+e^{m i \psi} \dot{v}=F^{q}\left(\ell \psi, e^{m i \psi} v\right) .
\end{gathered}
$$

Comparing the $y$-component with the $y$-component in (5.6), we have

$$
e^{-i \psi} F^{p}\left(\ell \psi, e^{m i \psi} v\right)=g(v)
$$

independent of $\psi$. Setting $\psi=0$ yields the relation $g(v)=F^{p}(0, v)$. The remaining relations are verified similarly. 
Not every system of equations of the form (5.6) arises as the pull-back of a system (5.3) on the center bundle: the pull-back equations are additionally equivariant with respect to an action of the isotropy subgroup $\mathbf{Z}_{\ell}$. It follows from the following lemma that the full group of symmetries of the pull-back equations is a semi-direct product of $\mathbf{S E}(2)$ and $\mathbf{Z}_{\ell}$.

Lemma 5.5 The pull-back equations are equivariant under the action (5.5) of $\mathbf{S E}(2)$ and under the following action of $\mathbf{Z}_{\ell}$ :

$$
(y, \psi, v) \mapsto\left(e^{2 \pi i / \ell} y, \psi, e^{2 \pi i m / \ell} v\right)
$$

There are no further restrictions on the pull-back equations.

Proof We have already established that the pull-back equations are SE(2)-equivariant under the action (5.5) and hence have the form (5.6). Next, we verify the $\mathbf{Z}_{\ell^{-} \text {-equivariance }}$ for the $G^{v}$-component:

$$
\begin{aligned}
G^{v}(v) & =F^{q}(0, v)-\frac{m i}{\ell} v F^{\varphi}(0, v)=F^{q}(2 \pi, v)-\frac{m i}{\ell} v F^{\varphi}(2 \pi, v) \\
& =e^{-2 \pi m i / \ell} F^{q}\left(0, e^{2 \pi m i / \ell} v\right)-\frac{m i}{\ell} v F^{\varphi}\left(0, e^{2 \pi m i / \ell} v\right) \quad(\text { by }(5.4)) \\
& =e^{-2 \pi m i / \ell} G^{v}\left(e^{2 \pi m i / \ell} v\right)
\end{aligned}
$$

The verification of (5.7) for the remaining components is similar.

Conversely, suppose that we are given a system of equations on $\mathbf{S E}(2) \times V_{0}$ that is equivariant under the actions $(5.5,5.7)$. By $\mathrm{SE}(2)$-equivariance, we can write the equations in the form (5.6) where $g, G^{\psi}, G^{v}$ depend on $v$. Define

$$
\begin{aligned}
& F^{p}(\varphi, q)=e^{i \varphi / \ell} g\left(e^{-i m \varphi / \ell} q\right) \\
& F^{\varphi}(\varphi, q)=\ell G^{\psi}\left(e^{-i m \varphi / \ell} q\right) \\
& F^{q}(\varphi, q)=e^{i m \varphi / \ell} G^{v}\left(e^{-i m \varphi / \ell} v\right)+m i v G^{\psi}\left(e^{-i n \varphi / \ell} v\right) .
\end{aligned}
$$

Then the system on $\mathrm{SE}(2) \times V_{0}$ is the pull-back of the system defined by (5.8). It remains to show that (5.8) is well-defined and equivariant under the action (3.2). Again concentrating on the third component, note that $F^{q}$ is well-defined if and only if

$$
F^{q}(\varphi+2 \pi, q)=F^{q}(\varphi, q) .
$$

But this equality follows from the $\mathbf{Z}_{\ell}$ equivariance of $G^{\psi}$ and $G^{v}$. Similarly, it is easily checked from definition (5.8) that $F^{q}$ satisfies the required $\mathrm{SE}(2)$-equivariance condition. 
This completes the first stage of the proof of Theorem 5.3. Next, we analyze the pull-back equations, emulating Theorem 4.2. Let $v(t)$ be a $2 \pi / \tilde{\omega}_{2}$ periodic solution to $\dot{v}=G^{v}(v)$. We suppose that this periodic solution is a result of Hopf bifurcation in the $\dot{v}$ equation. Define $\tilde{\omega}_{1}=G^{\psi}(v(0))$. As in Subsection 4.2, we solve $\dot{\psi}=G^{\psi}(v(t))$ for $\psi(t)$ and $\dot{y}=e^{i \psi(t)} g(v(t))$ for $y(t)$ to obtain a solution $(y(t), \psi(t), v(t))$.

Theorem 5.6 Let $(y(t), \psi(t), v(t))$ be the solution constructed above for the pull-back equations (5.6). Generically, $y(t)$ undergoes unbounded resonant growth if and only if

$$
\tilde{\omega}_{1}+k \tilde{\omega}_{2}=0
$$

for some integer $k$ satisfying

$$
k m \equiv 1 \quad(\bmod \ell)
$$

Proof The condition (5.9) is immediate from Theorem 4.2. We show that the additional

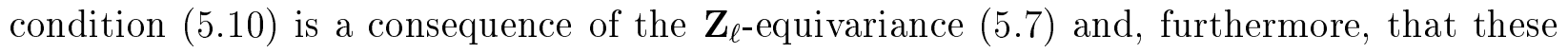
are the only restrictions on $k$.

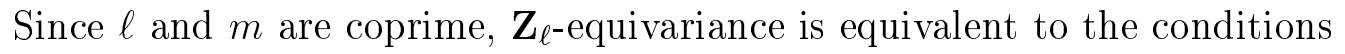

$$
\begin{aligned}
& \text { (a) } g\left(e^{2 \pi m i / \ell} v\right)=e^{2 \pi i / \ell} g(v) \\
& \text { (b) } G^{\psi}\left(e^{2 \pi i / \ell} v\right)=G^{\psi}(v) \\
& \text { (c) } G^{v}\left(e^{2 \pi i / \ell} v\right)=e^{2 \pi i / \ell} G^{v}(v) .
\end{aligned}
$$

It follows from (5.11)(c) and the uniqueness of periodic solutions in (generic) Hopf bifurcation that the periodic solution to the $\dot{v}$ equation satisfies

$$
v\left(t+\frac{2 \pi}{\ell \tilde{w}_{2}}\right)=e^{2 \pi i / \ell} v(t) .
$$

This observation is a special case of the spatio-temporal symmetries of symmetric Hopf bifurcation discussed in [8].

It follows from (5.12) and (5.11)(b) that solutions to the $\dot{\psi}$ equation have the form

$$
\psi(t)=\tilde{\omega}_{1} t+\tilde{\psi}(t)
$$

where $\tilde{\psi}$ is $\frac{2 \pi}{\ell \tilde{w}_{2}}$ periodic. Finally, using $(5.11)(\mathrm{a})$, we can rewrite the $\dot{y}$ equation as

$$
\dot{y}=e^{i \tilde{w}_{1} t} h(t)
$$


where $h$ is $2 \pi / \tilde{w}_{2}$ periodic and

$$
h\left(t+\frac{2 m \pi}{\ell \tilde{\omega}_{2}}\right)=e^{2 \pi i / \ell} h(t) .
$$

Write $h$ as a Fourier series

$$
h(t)=\sum_{k} h_{k} e^{i k \tilde{\omega}_{2} t}
$$

It follows from (5.13) that $h_{k}=0$ unless $k$ satisfies condition (5.10). In particular, these are the only values of $k$ for which the resonance (5.9) is possible.

Finally, we write down pull-back equations that exhibit the required resonances. Set $G^{\psi}(v)=\tilde{\omega}_{1}$ and $G^{v}(v)=i \tilde{\omega}_{2} v$. These functions clearly satisfy conditions (5.11)(b,c) and yield a $2 \pi / \tilde{\omega}_{2}$ periodic solution $v(t)$. The function $g(v)=v^{k}$ satisfies conditions (5.11)(a) provided $k$ satisfies condition (5.10) and yields those resonances (5.9) for which $k \geq 0$. To obtain the remaining resonances, consider $g(v)=\bar{v}^{k}$.

The third stage of the proof of Theorem 5.3 is to relate the frequencies $\tilde{\omega}_{j}$ in the pull-back equations with the frequencies $\omega_{j}$ in the original equations on the center bundle. Recall that the periodic solutions in the $\dot{v}$ equations arise via Hopf bifurcation and hence the frequencies $\tilde{\omega}_{j}(\lambda)$ vary smoothly with the bifurcation parameter $\lambda$. As in Subsection 4.3, we redefine $\tilde{\omega}_{j}=\tilde{\omega}_{j}(0)$. Then Theorem 5.6 implies that resonant growth occurs when

$$
\tilde{\omega}_{2} \sim-\frac{1}{k} \tilde{\omega}_{1}
$$

for some integer $k$ satisfying condition (5.10).

It follows from Proposition 5.4 that the frequencies $\tilde{\omega}_{j}$ and $\omega_{j}$ are related as follows:

$$
\tilde{\omega}_{1}=G^{\psi}(0)=\frac{1}{\ell} F^{\varphi}(0,0)=\frac{1}{\ell} \omega_{1}
$$

and

$$
i \tilde{\omega}_{2}=\left(d G^{v}\right)_{0}=\left(d_{q} F^{q}\right)_{0,0}-\frac{m i}{\ell} F^{\varphi}(0,0)=i \omega_{2}-\frac{m i}{\ell} \omega_{1} .
$$

Substituting these expressions into condition (5.14) yields the resonance criterion

$$
\omega_{2} \sim \frac{k m-1}{\ell k} \omega_{1}
$$

where $k$ satisfies (5.10).

Finally, we observe that equation (5.10) always has solutions for $\ell$ and $m$ coprime. If we let $m_{0}$ be the smallest positive integer $k$ satisfying (5.10), then, since $\ell$ and $m$ are coprime, all solutions to (5.10) have the form $k=j \ell+m_{0}$ for some integer $j$. This completes the proof of Theorem 5.3. 


\section{Takens-Bogdanov Bifurcation}

As mentioned previously, our approach can be applied to study other bifurcations from spiral waves. To illustrate this point, we consider two other bifurcations from one-armed spirals: steady-state bifurcation and the codimension two Takens-Bogdanov bifurcation. By Corollary 3.2, the associated center bundle will be a trivial bundle (this is not necessarily the case for these bifurcations from a many armed spiral).

Steady-state bifurcation leads to a saddle-node (or limit point) bifurcation of rotating wave spiral solutions with frequency close to $\omega_{1}$. We omit the details and pass to the more interesting Takens-Bogdanov bifurcation. In this case, the operator $(d \widetilde{\mathcal{F}})_{x_{0}, 0}$ in Subsection 4.3 has a nonsemisimple double zero eigenvalue and the corresponding center subspace $V_{0}$ is identified with $\mathbf{R}^{2}$. Let $q=(x, y) \in \mathbf{R}^{2}=V_{0}$. In normal form, the $\dot{q}$ equation in (4.1) can be written as

$$
\begin{aligned}
& \dot{x}=y \\
& \dot{y}=\mu_{1}+\mu_{2} y+x^{2}+b x y,
\end{aligned}
$$

where $\mu_{1}$ and $\mu_{2}$ are unfolding parameters and $b= \pm 1$ (cf. [9]). We consider the case $b=-1$, since it leads to stable limit cycles in (6.1).

A schematic of the phase portraits corresponding to regions in the unfolding space is given in Figure 5. Of particular interest is region A which is bounded by a curve of Hopf bifurcations and a curve of homoclinic bifurcations. Consider a path $P_{1}$ through this region. As $P_{1}$ crosses into region $\mathrm{A}$, there is a Hopf bifurcation from one of the equilibria. The resulting periodic solution of (6.1) generates quasiperiodic motion for the full system (4.1). As the homoclinic bifurcation curve is approached, the period of the periodic solution gets larger and tends to infinity (that is, its frequency gets smaller and tends to zero). Hence $P_{1}$ crosses an infinite number of resonance points. Consequently, in parameter space, $p(t)$ will experience unbounded growth for an infinite number of parameter values along $P_{1}$. However, as mentioned in the previous section, only the first one or two resonances should exhibit a well-defined change in petality in addition to unbounded growth.

Finally, consider a path $P_{2}$ through the saddle-node variety $\mu_{1}=0$. The interpretation of this bifurcation is the following. In region $\mathrm{B}$, a spiral wave is observed. As $P_{2}$ crosses into region $\mathrm{C}$, the spiral wave disappears and the dynamics enters a part of phase space not modeled by the center manifold equations. 


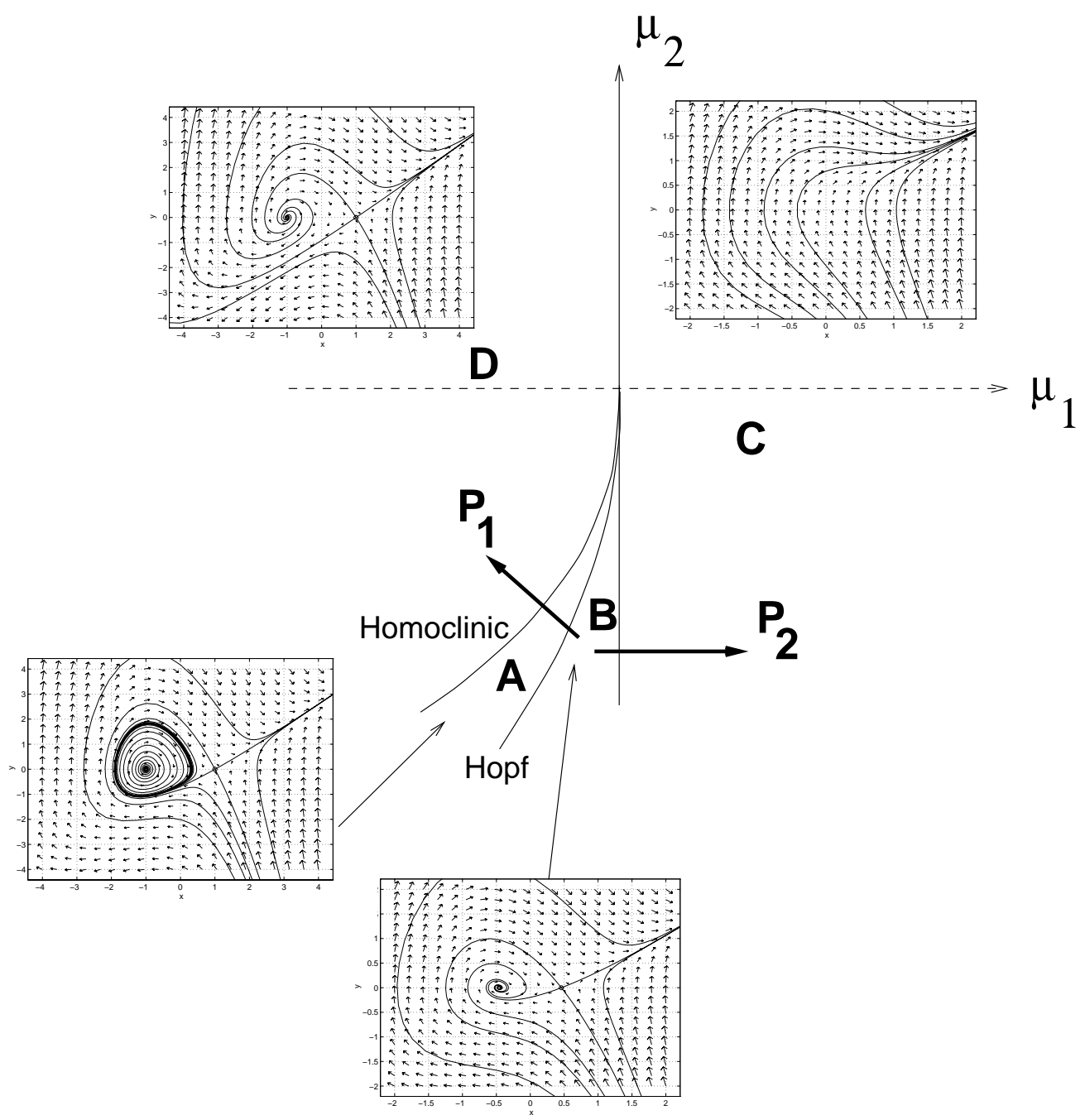

Figure 5: Unfolding of Takens-Bogdanov singularity in (6.1). 


\section{A Appendix}

In recent work, Sandstede et al. $[17,18]$ have generalized the center bundle construction of Krupa [12], described in Section 2, from compact groups to noncompact groups and from finite dimensions to infinite dimensions. In particular, they prove Conjecture 4.3 and provide a rigorous justification of the techniques in this paper. A major technical difficulty overcome in the work of $[21,17,18]$ is the lack of smoothness of the action of $\mathbf{S E}(2)$ on functions $u: \mathbf{R}^{2} \rightarrow \mathbf{R}$.

Independently, we have obtained a simplified reduction under the assumption (S1), see below, that the action of the Lie algebra of the group is 'smoother' than the linearized vector field defining the dynamical system. This assumption fails for actions of $\mathbf{S E}(2)$ but is satisfied in many important cases. Examples include the group $\mathbf{S O}(2)$ acting on $L^{2}(\Omega)$ for a circular bounded domain $\Omega$, and the noncompact group of translations $\mathbf{T}(2) \cong \mathbf{R}^{2}$ inside of $\mathbf{S E}(2)$ acting on $L^{2}\left(\mathbf{R}^{2}\right)$, see Example A.1. Hence our reduction, which is presented in this appendix, can be viewed as a rigorous and nontrivial extension of the methods of [12]. At the same time, our reduction hints at the full picture for noncompact group actions without addressing the technical difficulties resolved by $[21,17,18]$.

Under assumption (S1), we obtain the required decomposition $f=f_{N}+f_{T}$ into normal and tangent vector fields and we obtain the factorization (2.2) into normal dynamics coupled with drift along group orbits. To obtain a smooth center bundle, it is necessary that the critical eigenfunctions for the normal vector field are acted upon smoothly by the group, see assumption (S2). (It turns out that assumption (S2) is automatically satisfied for reactiondiffusion equations, see Sandstede et al. [17, 18].)

Throughout the appendix, smooth means $C^{k}$ for $k$ sufficiently large. We do not require that the group acts smoothly (or even continuously) on the whole of the infinite-dimensional space.

\section{A.1 The generalized center bundle reduction}

Let $\Gamma$ be a finite dimensional Lie group (not necessarily compact) acting by unitary transformations on a Hilbert space $\mathcal{H}$. Let $x_{0} \in \mathcal{H}$. We assume that the group orbit $X=\Gamma x_{0}$ is a smoothly embedded submanifold of $\mathcal{H}$. Let $\pi: N(X) \rightarrow X$ denote the normal bundle in $\mathcal{H}$, with fibers $N_{x}=\left(T_{x} X\right)^{\perp}$. Write points in $N(X)$ as $(x, v)$ where $x \in X$ and $v \in N_{x}$. The $\operatorname{map} \beta: N(X) \rightarrow \mathcal{H}, \beta(x, v)=x+v$ is a local diffeomorphism.

Since the action of $\Gamma$ is unitary, $N(X)$ is invariant under the action $(x, v) \rightarrow(\gamma x, \gamma v)$ and $\beta: N(X) \rightarrow \mathcal{H}$ is $\Gamma$-equivariant. Since $\beta$ is one-to-one on the homogeneous space $X$, it follows that $\beta$ restricts to a diffeomorphism on some $\Gamma$-invariant neighborhood of $X$. 
The dynamical system We suppose that $f: \mathcal{H} \rightarrow \mathcal{H}$ is a $\Gamma$-equivariant 'infinite dimensional vector field' on $\mathcal{H}$ (a nonlinear partial differential operator say). Suppose that $f$ satisfies the usual technical conditions so as to generate a smooth local semiflow on $\mathcal{H}$. That is, $f=A+N$ where the linearity $A$ is sectorial and the nonlinearity $N$ is sufficiently smooth on the domain $\mathcal{H}^{\alpha}$ of a fractional power $A^{\alpha}$ of $A$ for some $\alpha \in[0,1)$ see Henry [10] for the precise definitions). We note that $\mathcal{H}^{\alpha}$ is a dense and $\Gamma$-invariant subspace of $\mathcal{H}$. The graph norm $\|u\|_{\alpha}=\|u\|+\left\|A^{\alpha} u\right\|$ makes $\mathcal{H}^{\alpha}$ into a Hilbert space and $f$ is smooth when regarded as an operator $f: \mathcal{H}^{\alpha} \rightarrow \mathcal{H}$.

We suppose from now on that $\alpha$ has been chosen with these properties. Roughly speaking, $\alpha$ quantifies the 'semilinearity' of $f$ where the nonlinearity $N$ is strictly smoother than $A$ (so $\alpha$ is required to be strictly less than 1 ).

The Lie algebra The Lie algebra $L(\Gamma)$ consists of linear operators $\xi: \mathcal{H} \rightarrow \mathcal{H}$ defined by $\xi u=\left.\frac{d}{d t} \gamma_{t} u\right|_{t=0}$ where $\gamma_{t} \in \Gamma$ is a curve at the identity. Typically, the operators $\xi$ are unbounded and hence are not defined on the whole of $\mathcal{H}$. We let $\widetilde{\mathcal{H}}$ denote the common domain of the elements of $L(\Gamma)$ and note that $\widetilde{\mathcal{H}}$ is $\Gamma$-invariant. It follows from our assumption on $X$ that $X \subset \widetilde{\mathcal{H}}$.

Recall that $\alpha \in[0,1)$ is chosen so that $f: \mathcal{H}^{\alpha} \rightarrow \mathcal{H}$ is smooth. It is natural to make the simplifying assumption

(S1) $\mathcal{H}^{\alpha} \subset \widetilde{\mathcal{H}}$.

In particular, each infinitesimal generator $\xi \in L(\Gamma)$ is bounded as an operator $\xi: \mathcal{H}^{\alpha} \rightarrow \mathcal{H}$. (Roughly speaking, the Lie algebra elements are strictly smoother than the linear vector field $A$.)

Under assumption (S1), we obtain a complete generalization of the results in [12]. Theorems A.2 and A.6 correspond to [12, Theorems 2.1 and 2.2].

Example A.1 Suppose that $\mathcal{H}=L^{2}\left(\mathbf{R}^{2}\right)$ consists of functions $u: \mathbf{R}^{2} \rightarrow \mathbf{R}$ and that the group $\Gamma=\mathbf{S E}(2)$ acts by $u(x) \mapsto u\left(\gamma^{-1} x\right)$. A calculation shows that $L(\mathbf{S E}(2))$ is generated by

$$
\xi_{1} u=\frac{\partial u}{\partial x_{1}}, \quad \xi_{2} u=\frac{\partial u}{\partial x_{2}}, \quad \xi_{3} u=x_{1} \frac{\partial u}{\partial x_{2}}-x_{2} \frac{\partial u}{\partial x_{1}} .
$$

Suppose that the linear part $A$ of the evolution operator $f$ is the Laplacian $A=\Delta$. The generators $\xi_{1}, \xi_{2}$ corresponding to translation have less derivatives than $A$ and are relatively bounded with respect to $A$. In particular, we can choose $\alpha \in[1 / 2,1)$ in assumption (S1). However, the generator $\xi_{3}$ corresponding to rotation includes multiplication by the unbounded functions $x_{1}$ and $x_{2}$ and hence violates assumption (S1) for all $\alpha$. 
Note that the group of translations $\mathbf{T}(2) \cong \mathbf{R}^{2}$ satisfies assumption (S1). In addition, the rotation group $\mathbf{S O}(2)$ satisfies (S1) provided we restrict to $L^{2}(\Omega)$ for $\Omega$ a bounded circularly symmetric subset of $\mathbf{R}^{2}$.

In the remainder of this subsection we suppose that $x_{0}$ has discrete isotropy subgroup $\Sigma$. (This assumption is relaxed in Subsection A.2 below.)

Theorem A.2 Suppose that assumption (S1) is valid. Suppose further that $X=\Gamma x_{0}$ is a submanifold of $\mathcal{H}$ and that the isotropy subgroup $\Sigma$ of $x_{0}$ is discrete. There is a $\Gamma$-invariant neighborhood $U$ of $X=\Gamma x_{0}$ in $D_{A}$ and smooth $\Gamma$-equivariant nonlinear operators $f_{N}, f_{T}$ : $U \rightarrow \mathcal{H}$ such that

(i) $\left.f\right|_{U}=f_{N}+f_{T}$

(ii) $f_{N}(u) \in N_{\pi(u)}$ for all $u \in U$, and

(iii) $f_{T}(u) \in T_{u} \Gamma u$.

Proof For $u \in X$ we trivially have $\mathcal{H}=N_{\pi(u)} \oplus T_{u} \Gamma u$. Observe that this splitting remains valid for $u$ close to $X$ in the graph norm since $T_{u} \Gamma u=L(\Gamma) u$ varies smoothly with respect to this norm. Now define $f_{N}(u)$ and $f_{T}(u)$ by projecting $f(u)$ onto $N_{\pi(u)}$ and $T_{u} \Gamma u$ respectively. Again, the subspaces $N_{\pi(u)}$ and $T_{u} \Gamma u$ vary smoothly with $u$ and hence the associated projections are smooth.

The operators $f_{N}$ and $f_{T}$ are called the normal and tangent components of $f$. Note that $f_{T}$ is tangent to group orbits everywhere but $f_{N}$ is normal to group orbits only when restricted to $X$. Let $g=\left.f\right|_{N_{x_{0}} \cap U}$ denote the restriction of the normal operator to the normal fiber over $x_{0}$. Observe that $g$ is $\Sigma$-equivariant.

The standard results relating $(d g)_{x_{0}}$ with $(d f)_{x_{0}}$ are valid in this general setting, as we now describe. Suppose that $X$ is a relative equilibrium, so $f\left(x_{0}\right)=\xi x_{0}$ where $\xi \in L(\Gamma)$. Define $\tilde{f}(u)=f(u)-\xi u$, so that $x_{0}$ is an equilibrium for $\tilde{f}$. Then $(d \tilde{f})_{x_{0}}$ is a linear operator on $T_{x_{0}} N(X)=N_{x_{0}} \oplus T_{x_{0}} X \cong N_{x_{0}} \oplus L(\Gamma)$. Moreover, $(d \tilde{f})_{x_{0}}$ is sectorial, since $(d f)_{x_{0}}$ is sectorial and $\xi$ is defined on the domain of a fractional power.

Proposition A.3 Regarded as an operator on $N_{x_{0}} \oplus L(\Gamma),(d \tilde{f})_{x_{0}}$ has the form

$$
(d \tilde{f})_{x_{0}}=\left(\begin{array}{cc}
(d g)_{x_{0}} & 0 \\
\star & -\operatorname{ad}(\xi)
\end{array}\right) .
$$


Proof Restricting to the dense subspace $\widetilde{\mathcal{H}} \subset \mathcal{H}$ ensures that the mappings $f, g$ and so on are smooth. Recall that $f$ has the decomposition $f=f_{N}+f_{T}$ into normal and tangent components, so $\tilde{f}$ has the corresponding decomposition $\tilde{f}=f_{N}+\tilde{f}_{T}$ where $\tilde{f}_{T}(u)=f_{T}(u)-\xi u$. We show first that

$$
\left(d f_{N}\right)_{x_{0}}=\left(\begin{array}{cc}
(d g)_{x_{0}} & \star \\
\star & \star
\end{array}\right), \quad\left(d \tilde{f}_{T}\right)_{x_{0}}=\left(\begin{array}{cc}
0 & 0 \\
\star & \star
\end{array}\right)
$$

thus verifying the entries in the first column of $(d \tilde{f})_{x_{0}}$.

The form of $\left(d f_{N}\right)_{x_{0}}$ follows from the definition $g=\left.f_{N}\right|_{N_{x_{0}}}$. Now recall that $f_{T}(u)=$ $Q(u) f(u)$ where $Q(u): \mathcal{H} \rightarrow \mathcal{H}$ is the projection onto $T_{u} \Gamma u$ with kernel $N_{\pi(u)}$. Since $\xi u \in$ $T_{u} \Gamma u$, we have $\tilde{f}_{T}(u)=Q(u) \tilde{f}(u)$. For all $w \in T_{x_{0}} N(X)$, we compute that

$$
\left(d \tilde{f}_{T}\right)_{x_{0}} w=\left((d Q)_{x_{0}} w\right) \tilde{f}\left(x_{0}\right)+Q\left(x_{0}\right)(d \tilde{f})_{x_{0}} w=Q\left(x_{0}\right)(d \tilde{f})_{x_{0}} w \in T_{x_{0}} X
$$

since $\tilde{f}\left(x_{0}\right)=0$. Hence $\left(d \tilde{f}_{T}\right)_{x_{0}}$ has the required form.

It remains to verify the entries in the second column of $(d \tilde{f})_{x_{0}}$. Let $\eta x_{0}=\left.\frac{d}{d s} \gamma(s) x_{0}\right|_{s=0} \in$ $T_{x_{0}} X$ and observe that

$$
(d f)_{x_{0}} \eta x_{0}=\left.(d f)_{x_{0}} \frac{d}{d s} \gamma(s) x_{0}\right|_{s=0}=\left.\frac{d}{d s} f\left(\gamma(s) x_{0}\right)\right|_{s=0}=\left.\frac{d}{d s} \gamma(s) f\left(x_{0}\right)\right|_{s=0}=\eta f\left(x_{0}\right)=\eta \xi x_{0} .
$$

Hence,

$$
(d \tilde{f})_{x_{0}} \eta x_{0}=\left((d f)_{x_{0}}-\xi\right) \eta x_{0}=(\eta \xi-\xi \eta) x_{0}=-\operatorname{ad}(\xi)(\eta) x_{0} .
$$

Identifying $T_{x_{0}} X$ with $L(\Gamma)$, we have $(d \tilde{f})_{x_{0}} \eta=-\operatorname{ad}(\xi) \eta$ as required.

Remark A.4 It follows that modulo the eigenvalues of $-\operatorname{ad}(\xi)$, the spectrum of $(d g)_{x_{0}}$ coincides with the (relatively computable) spectrum of $(d \tilde{f})_{x_{0}}$. The eigenvalues of $-\operatorname{ad}(\xi)$ are viewed as neutral. Indeed, when $\Gamma$ is abelian we have $\operatorname{ad}(\xi) \equiv 0$.

When $\Gamma$ is compact, the adjoint action of $\Gamma$ preserves an inner product and it follows that $\operatorname{ad}(\xi)$ is a skew-symmetric matrix. In particular, the eigenvalues of $-\operatorname{ad}(\xi)$ are purely imaginary. This is the case also for noncompact groups, provided that the trajectory through $x_{0}$ is compact (in other words, the closure of $\{\exp t \xi, t \in \mathbf{R}\}$ is a compact subgroup of $\Gamma$ ).

In general, however, the eigenvalues of $-\operatorname{ad}(\xi)$ need not be purely imaginary. A somewhat hypothetical example is provided by the group of matrices

$$
\Gamma=\left\{\left(\begin{array}{ll}
a & b \\
0 & 0
\end{array}\right), a, b \in \mathbf{R}, a \neq 0\right\} .
$$


A basis for the Lie algebra is given by

$$
\xi_{1}=\left(\begin{array}{ll}
1 & 0 \\
0 & 0
\end{array}\right), \quad \xi_{2}=\left(\begin{array}{ll}
0 & 1 \\
0 & 0
\end{array}\right),
$$

and we calculate that $\operatorname{ad}\left(\xi_{1}\right)$ has eigenvalues 0 and 1 .

Corollary A.5 The normal vector field g generates a smooth local semiflow on $N_{x_{0}}$.

Proof Recall that $(d \tilde{f})_{x_{0}}$ is sectorial. By Proposition A.3, the spectra of $(d \tilde{f})_{x_{0}}$ and $(d g)_{x_{0}}$ differ by finitely many eigenvalues of finite multiplicity. Substituting vectors of the form $(\star, 0)$ into the formula (A.1), we obtain the estimate

$$
\left.\|(d g)_{x_{0}}-\mu\right)^{-1}\|\leq\|\left((d \tilde{f})_{x_{0}}-\mu\right)^{-1} \|,
$$

for all $\mu$ in the resolvent of $(d \tilde{f})_{x_{0}}$. Hence, sectoriality of $(d \tilde{f})_{x_{0}}$ implies sectoriality of $(d g)_{x_{0}}\left[10\right.$, Definition 1.3.1]. The domain $D_{(d g)_{x_{0}}}$ is given by $D_{(d g)_{x_{0}}}=D_{(d f)_{x_{0}}} \cap N_{x_{0}}$ and the nonlinearities in $g$ are as smooth as those in $f$, hence $g$ generates a smooth local semiflow.

Theorem A.6 Suppose that the hypotheses of Theorem A.2 and Corollary A.5 are valid. Let $u(t)$ and $v(t)$ be the solution trajectories for the initial value problems defined by the vector fields $f$ and $g$ with initial condition $v_{0} \in N_{x_{0}}$. Then, there is a smooth curve at the identity $\gamma(t) \in \Gamma$ such that

$$
u(t)=\gamma(t) v(t)
$$

Proof Since $f_{T}(v(t)) \in L(\Gamma) v(t)$ and $\Sigma$ is discrete, we can uniquely write $f_{T}(v(t))=$ $\xi(t) v(t)$ for a smooth curve $\xi(t) \in L(\Gamma)$. Let $\gamma(t)$ be the solution to the initial value problem $\frac{d}{d t} \gamma=\gamma \xi, \gamma(0)=e$. Then $\gamma(t)$ is a smooth curve at the identity as required.

Define $\tilde{u}(t)=\gamma(t) v(t)$. We show that $\tilde{u}(t)$ is a solution for the vector field $f$. It follows by uniqueness of solutions that $\tilde{u}=u$ and hence that $u=\gamma v$.

The solution $v(t)$ lies inside $\mathcal{H}^{\alpha} \cap N_{x_{0}}$ for $t>0$ and is smooth as a function $(0, \infty) \rightarrow \mathcal{H}^{\alpha}$ where defined (see [10, Sections 3.3 and 3.4]). It follows from assumption (S1) that $\tilde{u}(t)$ is $a$ priori at least once differentiable as an $\mathcal{H}$-valued map for $t>0$. (A postieri, $\tilde{u}(t)=u(t)$ is smooth as an $\mathcal{H}^{\alpha}$-valued map.) Applying the chain rule, we compute that

$$
\begin{aligned}
\frac{d}{d t} \tilde{u}(t) & =\frac{d}{d t} \gamma(t) v(t)=\gamma(t) \frac{d}{d t} v(t)+\left.\frac{d}{d s} \gamma(t+s) v(t)\right|_{s=0} \\
& =\gamma(t) g(v(t))+\gamma(t) \xi(t) v(t) \\
& =\gamma(t) f_{N}(v(t))+\gamma(t) f_{T}(v(t)) \\
& =\gamma(t) f(v(t))=f(\tilde{u}(t)
\end{aligned}
$$


It follows from Theorem A.6 that bifurcation from a relative equilibrium can be understood abstractly in terms of bifurcation from an equilibrium for the $\Sigma$-equivariant evolution operator $g$ coupled with drifts along the group.

If the spectrum of the linearization $(d g)_{x_{0}}$ of the normal vector field intersects the imaginary axis, and the remainder of the spectrum is bounded away from the imaginary axis, then the center manifold theorem applies on $N_{x_{0}}$. Since the action of $\Gamma$ is unitary, the norm on $\mathcal{H}$ is smooth and $\Gamma$-invariant. Hence, we can choose a smooth $\Gamma$-invariant 'cut-off' function $\chi: \mathcal{H} \rightarrow \mathbf{R}$ and we can construct a unique smooth $\Sigma_{x}$-invariant center manifold $W_{x}$ for the cut-off normal vector field $\left.\chi f_{N}\right|_{N_{x}}$ on each fiber $N_{x} \cap \mathcal{H}^{\alpha}, x \in X$. By construction, $W_{\gamma x}=\gamma W_{x}$ and hence the resulting center bundle $W=\cup_{x \in X} W_{x}$ is $\Gamma$-invariant and at least once differentiable since $\Gamma$ acts differentiably on $\mathcal{H}^{\alpha}$. In addition, each $W_{x}$ is a local center manifold for $\left.f\right|_{N_{x}}$ so that $W$ is a local center bundle for $f$. In order to proceed as in the main part of the paper, we require that

(S2) $\Gamma$ acts smoothly on elements in the center manifold $W_{x_{0}}$ for the normal vector field $g$.

It follows from assumption (S2) that $W$ is a smooth bundle and that $\Gamma$ acts smoothly on $W$. (Again, we note that $[17,18]$ have recently shown that assumption (S2) is automatically satisfied for reaction-diffusion equations.)

\section{A.2 Reductive isotropy subgroups}

In this subsection, we show that the results of Section A.1, in particular Theorems A.2 and A.6, are valid for more general classes of isotropy subgroup $\Sigma$. We continue to assume that $\Gamma$ is a finite dimensional Lie group acting by unitary transformations on $\mathcal{H}$, that assumption (S1) is valid, and that $X=\Gamma x_{0}$ is a smooth submanifold of $\mathcal{H}$.

The proof of Theorem A.2 breaks down when $x_{0}$ has isotropy subgroup $\Sigma$ of positive dimension. For example, there may be points $u$ of lower dimensional isotropy near $x_{0}$ in which case $\operatorname{dim} T_{u} \Gamma u$ is not constant. This difficulty is present even for compact Lie groups acting on $\mathbf{R}^{n}$ and is the main technical difficulty overcome in Krupa [12]. We now use the ideas in [12] to enlarge the class of isotropy subgroups $\Sigma$ for which Theorems A.2 and Theorem A.6 are valid.

Recall that $\Sigma$ is reductive if $\Sigma$ has a faithful finite dimensional representation and every finite dimensional representation of $\Sigma$ is completely reducible (every $\Sigma$-invariant subspace has a $\Sigma$-invariant complement). In particular, compact groups are reductive. The next result generalizes [12, Lemma 2.3]. 
Lemma A.7 Suppose that assumption (S1) is valid, that $X=\Gamma x_{0}$ is a submanifold of $\mathcal{H}$, and that the isotropy subgroup $\Sigma$ of $x_{0}$ is reductive. There exists a smooth $\Gamma$-invariant subbundle $\left.K \subset T N(X)\right|_{\mathcal{H}^{\alpha}}$ such that for all $u$ in a $\Gamma$-invariant neighborhood $U \subset \mathcal{H}^{\alpha}$ of $X$,

(i) $K_{u} \subset T_{u} \Gamma u$, and

(ii) $N_{\pi(u)} \oplus K_{u}=\mathcal{H}$.

Proof The subspace $L(\Sigma) \subset L(\Gamma)$ is invariant under the adjoint action of $\Sigma$ and hence, by reductivity, there is an invariant subspace $Y \subset L(\Gamma)$ such that

$$
L(\Gamma)=L(\Sigma) \oplus Y .
$$

Let $u=\left(\gamma x_{0}, v\right) \in \mathcal{H}^{\alpha} \subset N(X)$ and define

$$
K_{u}=\left(\operatorname{Ad}_{\gamma} Y\right) u .
$$

Note that $K_{u}$ is well-defined due to the invariance of $Y$. For suppose that $\gamma_{1} x_{0}=\gamma_{2} x_{0}$. Then $\gamma_{2}^{-1} \gamma_{1} \in \Sigma$ and so $\operatorname{Ad}_{\gamma_{1}} Y=\operatorname{Ad}_{\gamma_{2}} Y$. Hence $K$ is a subbundle of $N(X)$ satisfying condition (i). In contrast to the tangent spaces $T_{u} \Gamma u$, the subspaces $K_{u}$ are of constant dimension near $X$ and condition (ii) is satisfied.

To show that $K$ is $\Gamma$-invariant, we prove that $K_{\gamma u}=\gamma K_{u}$. Indeed, if $u=\left(\delta x_{0}, v\right)$, then

$$
K_{\gamma u}=\left(\operatorname{Ad}_{\gamma \delta} Y\right) \gamma u=\left(\operatorname{Ad}_{\gamma} \operatorname{Ad}_{\delta} Y\right) \gamma u=\gamma\left(\operatorname{Ad}_{\delta} Y\right) u=\gamma K_{u} .
$$

Finally, smoothness of the bundle is proved as in [12].

It is now straightforward to extend Theorem A.2 from discrete isotropy subgroups to reductive isotropy subgroups. Simply project $f(u)$ onto $N_{\pi(u)}$ and $K_{u}$. Similarly, the curve $\xi(t)$ in the proof of Theorem A.6 is chosen to lie in $K_{v(t)}$. The remaining proofs in Section A.1 are unchanged.

Remark A.8 The assumptions underlying our generalization of Krupa's center bundle reduction can be summarized as follows. The simplifying assumption (S1) ensures that the generalization from finite dimensions to infinite dimensions runs smoothly. We require that the relative equilibrium $X=\Gamma x_{0}$ is a smooth embedded submanifold of $\mathcal{H}$. Assumption (S2) guarantees that the center bundle is smooth and not merely once differentiable.

Our results are proved for two classes of isotropy subgroups of $x_{0}: \Sigma$ discrete and $\Sigma$ reductive. Actually, we require only that there is an invariant complement for $L(\Sigma) \subset L(\Gamma)$ under the adjoint action of $\Sigma$ (this is trivially the case when $\Sigma$ is discrete even if $\Sigma$ is not reductive). An example where this property is violated is provided by the subgroup $\Sigma=\mathbf{T}(2)$ of translations in $\Gamma=\mathbf{S E}(2)$. We conjecture that there is no analogue of Theorems A.2 and A.6 in such cases. 


\section{Acknowledgments}

We wish to thank Dwight Barkley, Mike Field, Iannis Kevrekidis, Martin Krupa, Zhenya Nikolaev, Qi Ouyang, Ian Stewart, and Harry Swinney for helpful conversations concerning the meandering of spirals. In particular, we wish to thank Bernold Fiedler for his suggestion of how to analyze meandering Hopf bifurcations from multi-armed spirals. In addition, we thank the referees for a number of helpful remarks.

This research was supported in part by NSF Grant DMS-9403624 (MG, IM), ONR Grant N00014-94-1-0317 (MG, VGL, IM), the Texas Advanced Research Program (003652037) (MG), and an NSERC Post-Doctoral Fellowship (VGL). VGL also acknowledges the support of the Department of Mathematics of the University of Houston.

\section{References}

[1] D. Barkley. A model for fast computer-simulation of waves in excitable media. Physica D 49 (1991) 61-70.

[2] D. Barkley. Linear stability analysis of rotating spiral waves in excitable media. Phys. Rev. Lett. 68 (1992) 2090-2093.

[3] D. Barkley. Euclidean symmetry and the dynamics of rotating spiral waves. Phys. Rev. Lett. 72 (1994) 165-167.

[4] D. Barkley, M. Kness and L.S. Tuckerman. Spiral-wave dynamics in a simple model of excitable media: The transition from simple to compound rotation. Phys. Rev. A 42 (1990) 2489-2492.

[5] V.N. Biktashev, A.V. Holden and E.V. Nikolaev. Spiral wave meander and symmetry in the plane. Intern. J. Bifur. \& Chaos 6 (1996).

[6] B. Fiedler, B. Sandstede, A. Scheel and C. Wulff. Bifurcation from relative equilibria of noncompact group actions: skew products, meanders and drifts. Documenta Math $\mathbf{1}$ (1996) 479-505.

[7] M. J. Field. Equivariant dynamical systems. Trans. Amer. Math. Soc. 259 (1980) 185205.

[8] M. Golubitsky and I.N. Stewart. Hopf bifurcation in the presence of symmetry. Arch. Rational Mech. 85 Anal. 87 No. 2 (1985) 107-165. 
[9] J. Guckenheimer and P. Holmes. Nonlinear Oscillations, Dynamical Systems, and Bifurcations of Vector Fields. Appl. Math Sci. 42, Springer-Verlag, New York, 1983.

[10] D. Henry. Geometric Theory of Semilinear Parabolic Equations. Lecture Notes in Math. 840, Springer, Berlin, 1981.

[11] R. Kapral and K. Showalter. Chemical Waves and Patterns. Kluwer Academic Publishers, Amsterdam, 1995.

[12] M. Krupa. Bifurcations from relative equilibria, SIAM J. Math. Anal. 21 (1990) 14531486.

[13] W. Jahnke, W. E. Skaggs and A. T. Winfree. Chemical vortex dynamics in the BelousovZhabotinsky reaction and in the two-variable Oregonator model. J. Phys. Chem. 93 (1989) 740-749.

[14] G. Li, Q. Ouyang, V. Petrov and H. L. Swinney. Transition from simple rotating chemical spirals to meandering and traveling spirals. Phys. Rev. Lett. 77 (1996) 2105-2108.

[15] D. Rand. Dynamics and symmetry. Predictions for modulated waves in rotating fluids, Arch. Rational Mech. \& Anal. 79 (1982) 1-38.

[16] M. Renardy. Bifurcation from rotating waves, Arch. Rational Mech. \& Anal. 79 (1982) $49-84$.

[17] B. Sandstede, A. Scheel and C. Wulff. Center-manifold reduction for spiral waves. $C$. R. Acad. Sci. To appear.

[18] B. Sandstede, A. Scheel and C. Wulff. Dynamics of spiral waves in unbounded domains using center-manifold reductions. Preprint.

[19] A. Vanderbauwhede, M. Krupa and M. Golubitsky. Secondary bifurcations in symmetric systems. In: Proc. Equadiff Conference, 1987, (C.M. Dafermos, G. Ladas and G. Papanicolaou, Eds.) Lect. Notes in Pure \& Appl. Math., 118 Marcel Dekker, New York, 1989, 709-716.

[20] A.T. Winfree. Scroll-shaped waves of chemical activity in three dimensions, Science 181 (1973) 937-939.

[21] C. Wulff. Theory of Meandering and Drifting Spiral Waves in Reaction-Diffusion Systems. Freie Universität Berlin, Thesis, 1996. 